\title{
Effective long-distance interaction from short-distance interaction in a periodically driven one-dimensional classical system
}

\author{
Lingzhen Guo, ${ }^{1,2}$ Modan Liu, ${ }^{1,3}$ and Michael Marthaler ${ }^{1}$ \\ ${ }^{1}$ Institut für Theoretische Festkörperphysik, Karlsruhe Institute of Technology (KIT), D-76131 Karlsruhe, Germany \\ ${ }^{2}$ Department of Microtechnology and Nanoscience (MC2), Chalmers University of Technology, SE-41296 Göteborg, Sweden \\ ${ }^{3}$ Department of Physics, Beijing Normal University, Beijing 100875, China
}

(Received 14 March 2016; published 20 May 2016)

\begin{abstract}
We study the classical dynamics of many interacting particles in a periodically driven one-dimensional (1D) system. We show that under the rotating wave approximation (RWA), a short-distance 1D interaction ( $\delta$ function or hard-core interaction) becomes a long-distance two-dimensional (2D) interaction which only depends on the distance in the phase space of the rotating frame. The RWA interaction describes the effect of the interaction on the slowly changing amplitude and phase of the oscillating particles, while the fast oscillations take on the role of a force carrier, which allows for interaction over much larger effective distances.
\end{abstract}

DOI: 10.1103/PhysRevA.93.053616

\section{INTRODUCTION}

The properties of one-dimensional (1D) interacting particles have been investigated as early as 1936 by Tonks [1]. Later in the 1960s, Tonk's model was extended to the more general Lieb-Liniger model by allowing the strength of particles' contact interaction ( $\delta$ function interaction) to be arbitrary. The exact solution and the thermodynamics of the Lieb-Liniger model have been discussed extensively [2-5]. In the past two decades progress in the field of ultracold atoms has been significant [6-9] and possibilities to realize Lieb-Liniger model have been proposed [10-12]. Following these theoretical proposals, the Tonks gas and Lieb-Liniger gas were observed by several experimental groups [13-15]. Due to these advances, the properties of interacting particles in one dimension have gained renewed interest [16,17]. Beyond interacting particles in 1D free space, the properties of interacting particles in a 1D harmonic trap are also of great interest, both in theory [18-25] and in experiments [16,17,26].

Ultracold atoms under periodic driving are particularly useful and interesting since periodic driving allows for the generation of artificial gauge fields [9,27-31]. Similar to Bloch's theorem for spatial periodicity, the Floquet theory [32-36] can be used to treat time-periodic quantum systems. The Floquet method transforms a periodic time-dependent Schrödinger equation into an eigenvalue problem of a timeindependent Floquet Hamiltonian, which is more accessible to a theoretical treatment. An intriguing fact of a Floquet Hamiltonian is that it can be used to simulate time-independent Hamiltonians that are difficult to access otherwise. Additonally, the Floquet Hamiltonian allows for the study of novel phenomena going beyond equilibrium physics [37-44]. In the classical limit, the Floquet method corresponds to Poincaré mapping $[45,46]$, which means we observe the particles every fixed time period and mark them on the phase space according to their instantaneous positions and momenta. The particles' trajectories on phase space in the discrete time domain can be considered as stroboscopic dynamics. Most previous works using Floquet theory are based on the single-particle picture, i.e., neglecting the interaction between particles. Recent works $[47,48]$ realized the importance of particles' interaction in periodically driven systems and started to develop scattering theory for Floquet states.
In this work, we investigate the classical dynamics of interacting identical particles in a 1D harmonic trap in the presence of various types of interaction potentials, e.g., $\delta$ function interaction, Coulomb interaction, hard-core interaction, and Lennard-Jones interaction. The main goal is to determine how the particles' stroboscopic dynamics is influenced by their interaction potential $V\left(x_{i}-x_{j}\right)$. By going to a rotating frame and using the rotating wave approximation (RWA), we transform the $1 \mathrm{D}$ spatial interaction $V\left(x_{i}-x_{j}\right)$ to a $2 \mathrm{D}$ interaction in phase space $U\left(R_{i j}\right)$, which only depends on two particles' distance in phase space $R_{i j}$. However, there is a divergence problem in calculating the RWA interaction for, e.g., Coulomb interaction. We analyze the origin of divergence and introduce a renormalization procedure to obtain the correct RWA interaction. Interestingly, we find a short-distance (e.g., hard-core interaction) real-space interaction can generate a long-distance RWA interaction on phase space, which increases linearly with phase-space distance. We justify our renormalized RWA interaction by simulating the many-body dynamics numerically and compare the results to our theory.

The article is organized as follows. In Sec. II, we describe our model of interacting particles in a 1D harmonic trap under periodic driving. In Sec. III, we introduce the RWA Hamiltonian by transforming to the rotating frame. We derive the general expression to calculate the RWA interaction $U\left(R_{i j}\right)$ for a given real-space interaction $V\left(x_{i}-x_{j}\right)$. In Sec. IV, we give the canonical equations of motion corresponding to both the original Hamiltonian and the RWA Hamiltonian. To obtain the stroboscopic dynamics of interacting particles, we also dicuss the Poincaré mapping method. In Sec. V, we calculate several examples of RWA interactions. We point out the divergence problem appearing in the case of Coulomb interaction. Then we introduce the renormalization procedure to get the correct RWA interaction. We apply our renormalization method to a more general case of an inverse power-law interaction potential and the Lennard-Jones interaction potential. In Sec. VI, we investigate the two-body dynamics and the three-body dynamics with different types of interactions. We also justify our RWA interaction by showing the dynamics of eight interacting particles under driving. In Sec. VII, we summarize our work and give an outlook for future work. 


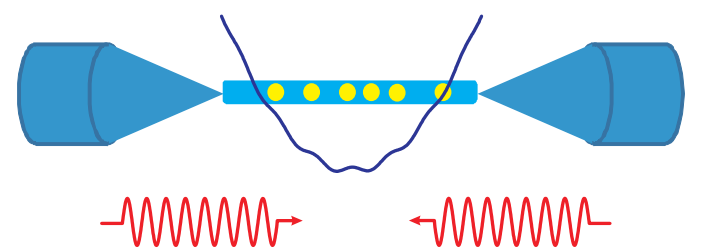

FIG. 1. Sketch of interacting particles in 1D harmonic trap. Ultracold atoms (yellow dots) are trapped in 1D harmonic potential. The atoms are further driven by two counterpropagating laser beams (red waves). The intensity of laser beams is tuned periodically, i.e., $\propto \cos \omega_{d} t$. The total potential at a fixed moment (blue rippled curve) is the harmonic trapping potential plus a cosine function. The interaction potential between atoms is assumed to be $V\left(x_{i}-x_{j}\right)$.

\section{MODEL}

We consider many identical particles confined in a 1D harmonic potential and driven by an external driving field as sketched in Fig. 1. The particles have the same mass $m$ and harmonic frequency $\omega$. For ultracold atoms, the driving field can be produced by the interference of two counterpropagating laser beams [6,7] with wavelength $a$. The intensity of laser beams is tuned periodically, i.e., $\propto \cos \left(\omega_{d} t\right)$. We assume the interaction potential between atoms is $V\left(x_{i}-x_{j}\right)$. The total Hamiltonian then is described by

$$
\begin{aligned}
H(t)= & \sum_{i}\left[\frac{p_{i}^{2}}{2}+\frac{x_{i}^{2}}{2}+\Lambda \cos (\Omega t) \cos \left(x_{i}\right)\right] \\
& +\sum_{i<j} V\left(x_{i}-x_{j}\right) .
\end{aligned}
$$

Here, the Hamiltonian has been scaled by the energy unit $m \omega^{2} a^{2} /\left(4 \pi^{2}\right)$. The time, coordinate, and momentum are scaled by $\omega^{-1}, a /(2 \pi)$, and $m \omega a /(2 \pi)$, respectively. We also introduced the scaled dimensionless driving frequency $\Omega \equiv \omega_{d} / \omega$ and driving strength $\Lambda \equiv 4 \pi^{2} f /\left(m \omega^{2} a^{2}\right)$. We define the detuning parameter via $\delta \equiv 1-\Omega / k$, where $k$ is a positive integer. In this paper, we work in the regime near the resonant condition, i.e., $|\delta| \ll 1$. Similar Hamiltonians can also be created using superconducting devices [49].

We are interested in the case of weak driving regime $\Lambda \ll 1$, which means we are not working on a periodic lattice model. In our present model, the harmonic trapping potential plays an important role and the Hamiltonian (1) does not have spatial periodicity. The basic motion of a particle is dominated by the global harmonic oscillation with frequency $\Omega / k$. The driving field and particles' interactions perturb the phase and amplitude of the global harmonic motion. Thus the total motion of a particle can be separated into a fast global oscillation with frequency $\Omega / k$ and a much slower motion representing the dynamics of the phase and amplitude of the global motion

$$
\left\{\begin{array}{l}
x_{i}(t)=P_{i}(t) \sin \left(\frac{\Omega}{k} t\right)+X_{i}(t) \cos \left(\frac{\Omega}{k} t\right), \\
p_{i}(t)=P_{i}(t) \cos \left(\frac{\Omega}{k} t\right)-X_{i}(t) \sin \left(\frac{\Omega}{k} t\right) .
\end{array}\right.
$$

The main task of this paper is to identify the role of interaction $V\left(x_{i}-x_{j}\right)$ on the slow dynamics of $X_{i}(t)$ and $P_{i}(t)$.

\section{RWA HAMILTONIAN}

We transform the original Hamiltonian (1) into the rotating frame with frequency $\Omega / k$ using the generating function of the second kind

$$
\begin{aligned}
G_{2}(\vec{x}, \vec{P}, t)= & \sum_{i} \frac{x_{i} P_{i}}{\cos (\Omega t / k)}-\frac{1}{2} x_{i}^{2} \tan \left(\frac{\Omega}{k} t\right) \\
& -\frac{1}{2} P_{i}^{2} \tan \left(\frac{\Omega}{k} t\right) .
\end{aligned}
$$

Here, $\vec{x}=\left(x_{1}, x_{2}, \ldots\right)$ and $\vec{P}=\left(P_{1}, P_{2}, \ldots\right)$ represent the assembled canonical coordinates of all the particles. The corresponding canonical transformations of coordinates and momenta are given by $p_{i}=\partial G_{2}(\vec{x}, \vec{P}, t) / \partial x_{i}, \quad X_{i}=$ $\partial G_{2}(\vec{x}, \vec{P}, t) / \partial P_{i}$, which result in the transformation (2). The canonical transformation of the Hamiltonian itself is given by $H(t)+\partial G_{2} / \partial t$. Using the rotating wave approximation (RWA), i.e., dropping fast oscillating terms in $H(t)+\partial G_{2} / \partial t$, we get the RWA Hamiltonian of all interacting particles in the rotating frame,

$$
g=\sum_{i}\left[\frac{1}{2} \delta r_{i}^{2}+\Lambda \cos \left(\frac{k \pi}{2}\right) J_{k}\left(r_{i}\right) \cos \left(k \theta_{i}\right)\right]+\sum_{i<j} U\left(R_{i j}\right) .
$$

Here, we have defined the vector displacement of the $i$ th particle in phase space by $r_{i} e^{i \theta_{i}} \equiv X_{i}+i P_{i}$ and two particles' phase-space distance by

$$
R_{i j} \equiv\left|r_{i} e^{i \theta_{i}}-r_{j} e^{i \theta_{j}}\right|=\sqrt{\left(X_{i}-X_{j}\right)^{2}+\left(P_{i}-P_{j}\right)^{2}} .
$$

The detailed derivations are given in Appendix A. The RWA approximation is valid for small detuning and weak driving, i.e., $|\delta| \ll 1$ and $\Lambda \ll 1$.

Normally, the original interaction potential $V\left(x_{i}-x_{j}\right)$ is only a function of the two particles' distance $\left|x_{i}-x_{j}\right|$, which implies $V(x)=V(-x)$. Given a particular type of interaction potential $V(x)$, we first define the Fourier transformation of the potential, i.e., $V_{q}=\frac{1}{2 \pi} \int_{-\infty}^{+\infty} d x V(x) e^{-i q x}$. Then the RWA interaction is given by (see more derivation details in Appendix A)

$$
U\left(R_{i j}\right)=\int_{-\infty}^{+\infty} d q V_{q} J_{0}\left(q R_{i j}\right)
$$

where $J_{0}(\bullet)$ is the Bessel function of zeroth order. Using the integral representation of the Bessel function, i.e., $J_{0}(x)=$ $\frac{1}{2 \pi} \int_{-\pi}^{+\pi} e^{-i x \sin \tau} d \tau$, we get an alternative form of the RWA interaction as follows:

$$
U\left(R_{i j}\right)=\frac{1}{2 \pi} \int_{0}^{2 \pi} V\left(R_{i j} \sin \tau\right) d \tau=\frac{2}{\pi} \int_{0}^{\frac{\pi}{2}} V\left(R_{i j} \sin \tau\right) d \tau .
$$

We see that $U\left(R_{i j}\right)$ is in fact the time average of the interaction energy over the oscillation period under RWA. Since the RWA interaction $U\left(R_{i j}\right)$ is defined in the phase space of the rotating frame and it is only a function of phase-space distance $R_{i j}$, we call it phase-space interaction. 


\section{CANONICAL EQUATIONS OF MOTION}

The time evolution of the original coordinates, $x_{i}(t)$ and $p_{i}(t)$, of a particle are described by the canonical equations of motion (EOM) according to the original Hamiltonian (1)

$$
\frac{d x_{i}}{d t}=\frac{\partial H(t)}{\partial p_{i}}, \quad \frac{d p_{i}}{d t}=-\frac{\partial H(t)}{\partial x_{i}} .
$$

As seen from transformation (2), the values of $X_{i}(t)$ and $P_{i}(t)$ can be obtained from the time evolution of $x_{i}(t)$ and $p_{i}(t)$ stroboscopically every time period of

$$
\begin{gathered}
\Delta t=\frac{2 k \pi}{\Omega} . \\
\qquad \begin{array}{l}
\dot{X}_{i}=\delta P_{i}+\Lambda \cos \left(\frac{k \pi}{2}\right)\left[J_{k}^{\prime}\left(r_{i}\right) \cos \left(k \theta_{i}\right) \frac{\partial r_{i}}{\partial P_{i}}\right. \\
\dot{P}_{i}=-\delta X_{i}-\Lambda \cos \left(\frac{k \pi}{2}\right)\left[J_{k}^{\prime}\left(r_{i}\right) \cos \left(k \theta_{i}\right) \frac{\partial r_{i}}{\partial X_{i}}\right.
\end{array} \\
\text { If we define a complex coordinate via } Z_{i} \equiv r_{i} e^{i \theta_{i}}=X_{i}+i P_{i}, \\
\text { the EOM (9) can be written in an alternative form as follows: } \\
\qquad \begin{array}{c}
d Z_{i}=-\left[\Lambda \cos \left(\frac{k \pi}{2}\right) \frac{k J_{k}\left(r_{i}\right)}{r_{i}^{2}} \sin \left(k \theta_{i}\right)\right] Z_{i} \\
-i\left[\delta+\Lambda \cos \left(\frac{k \pi}{2}\right) \frac{J_{k}^{\prime}\left(r_{i}\right)}{r_{i}} \cos \left(k \theta_{i}\right)\right] Z_{i}
\end{array} \\
-i \sum_{j} U^{\prime}\left(\left|Z_{i}-Z_{j}\right|\right) \frac{Z_{i}-Z_{j}}{\left|Z_{i}-Z_{j}\right|} .
\end{gathered}
$$$$
\left\{\begin{array}{l}
\dot{X}_{i}=\delta P_{i}+\Lambda \cos \left(\frac{k \pi}{2}\right)\left[J_{k}^{\prime}\left(r_{i}\right) \cos \left(k \theta_{i}\right) \frac{\partial r_{i}}{\partial P_{i}}-k J_{k}\left(r_{i}\right) \sin \left(k \theta_{i}\right) \frac{\partial \theta_{i}}{\partial P_{i}}\right]+\sum_{j} U^{\prime}\left(R_{i j}\right) \frac{P_{i}-P_{j}}{R_{i j}}, \\
\dot{P}_{i}=-\delta X_{i}-\Lambda \cos \left(\frac{k \pi}{2}\right)\left[J_{k}^{\prime}\left(r_{i}\right) \cos \left(k \theta_{i}\right) \frac{\partial r_{i}}{\partial X_{i}}-k J_{k}\left(r_{i}\right) \sin \left(k \theta_{i}\right) \frac{\partial \theta_{i}}{\partial X_{i}}\right]-\sum_{j} U^{\prime}\left(R_{i j}\right) \frac{X_{i}-X_{j}}{R_{i j}} .
\end{array}\right.
$$

Here $\Delta t$ is defined as the period of the stroboscopic dynamics. In this sense, the slow dynamics of $X_{i}(t)$ and $P_{i}(t)$ is defined in the discrete time domain $t=m \Delta t$ with $m=0, \pm 1, \pm 2, \ldots$. This technique is called Poincaré mapping.

In the rotating frame, the dynamics of $X_{i}(t)$ and $P_{i}(t)$ is described by the canonical EOM according to the RWA Hamiltonian (3), i.e.,

$$
\frac{d X_{i}}{d t}=\frac{\partial g}{\partial P_{i}}, \quad \frac{d P_{i}}{d t}=-\frac{\partial g}{\partial X_{i}} .
$$

From the relationship $r_{i} e^{i \theta_{i}}=X_{i}+i P_{i}$, we have the explicit form of the canonical EOM (8) as follows (see the details in Appendix B):
We see that the time evolution of $Z_{i}(t)$ is determined by three "forces." The right-hand-side term in the first line of Eq. (10) is the force parallel to $Z_{i}$ produced by driving. The second line of Eq. (10) is the force perpendicular to $Z_{i}$ produced by detuning and driving. The third line of Eq. (10) is the force produced by the interactions with other particles. The unit vector $\frac{Z_{i}-Z_{j}}{\left|Z_{i}-Z_{j}\right|}$ represents the direction from $j$ th particle to $i$ th particle. The interaction strength is proportional to the derivative of the RWA interaction, i.e., $U^{\prime}\left(\left|Z_{i}-Z_{j}\right|\right)=d U\left(\left|Z_{i}-Z_{j}\right|\right) / d\left|Z_{i}-Z_{j}\right|$. Different from the interaction in the laboratory, the imaginary unit $i$ appearing in this term indicates the direction of this interaction force is perpendicular to the line connecting two particles. Based on the EOM (9) or (10), we can calculate all the trajectories of interacting particles on phase space. The key of the above EOMs is to determine the explicit form of the RWA interaction $U\left(R_{i j}\right)$. Below, we will discuss some examples of interaction potentials $V\left(r_{i}-r_{j}\right)$ and calculate their corresponding RWA interactions $U\left(R_{i j}\right)$.

\section{RWA INTERACTIONS}

\section{A. Examples of interaction potentials}

In this subsection, we calculate the RWA interactions for several specific interaction potentials $V\left(x_{i}-x_{j}\right)$, i.e., the $\delta$ function interaction potential, the rectangular interaction potential, the hard-core interaction, and the Coulomb interaction potential. We point out the problem of divergence in the case of Coulomb interaction, which can be solved by the renormalization procedure introduced in the next subsection.

\section{1. $\delta$ function interaction potential}

The $\delta$ function interaction (contact interaction) is used to describe the effective interaction between neutral ultracold atoms in quasi-1D confinement [10-15]. We describe the $\delta$ function by a Lorenz function in the limiting case of vanishing width, i.e.,

$$
V\left(x_{i}-x_{j}\right)=\beta \delta\left(x_{i}-x_{j}\right)=\lim _{\varepsilon \rightarrow 0} \frac{\beta}{\pi} \frac{\varepsilon}{\left(x_{i}-x_{j}\right)^{2}+\varepsilon^{2}} .
$$

We introduce the Lorentz function here because we will later use it for the numerical simulation. Here, $\beta$ is the strength of the $\delta$ function interaction. The Fourier transformation coefficient of the above Lorentz function is $V_{k}=\beta e^{-|k| \varepsilon} /(2 \pi)$. One can obtain the RWA interaction from Eq. (4) or Eq. (5)

$$
U\left(R_{i j}\right)=\frac{\beta}{\pi} \frac{1}{\sqrt{R_{i j}^{2}+\varepsilon^{2}}} .
$$

In the limit of $\varepsilon \rightarrow 0$, we have the RWA interaction

$$
U\left(R_{i j}\right)=\frac{\beta}{\pi R_{i j}} .
$$

It is interesting to note that the short-distance $\delta$ interaction potential produces an effective long-distance Coulomb-like interaction as function of phase-space distance $R_{i j}$.

\section{Rectangular interaction potential}

The $\delta$ function interaction is a pointlike interaction with zero interaction range. Now we allow that the interaction has a finite range and define a rectangular interaction potential

$$
\eta \operatorname{rect}\left(\frac{x_{i}-x_{j}}{2 \beta}\right)= \begin{cases}0 & \text { if }\left|x_{i}-x_{j}\right|>\beta \\ \eta / 2 & \text { if }\left|x_{i}-x_{j}\right|=\beta \\ \eta & \text { if }\left|x_{i}-x_{j}\right|<\beta\end{cases}
$$

Here, $\beta$ is the interaction range and $\eta$ is the interaction strength. Applying formula (5), we have (see more details 
in Appendix C)

$$
U\left(R_{i j}\right)= \begin{cases}\frac{2 \eta}{\pi} \arcsin \left(\frac{\beta}{R_{i j}}\right) & \text { if } R_{i j} \geqslant \beta, \\ \eta & \text { if } R_{i j}<\beta .\end{cases}
$$

For $R_{i j} \gg \beta$, we have the long-range asymptotic behavior

$$
U\left(R_{i j}\right) \sim \frac{2 \eta \beta}{\pi} \frac{1}{R_{i j}}, \text { for } R_{i j} \gg \beta .
$$

Again, we have an effective long-distance Coulomb-type RWA interaction from a short-range real-space interaction.

\section{Hard-core interaction potential}

In the discussion of the rectangular interaction potential, we have assumed the potential height $\eta$ is finite. This means that the two particles can overcome the potential barrier and particles can pass each other if their relative kinetic energy is large enough. When $\eta$ is larger than the two particles' relative kinetic energy, the particles cannot overcome the barrier and thus are rebounded back. If the phase-space distance of two particles is $R_{i j}$, their relative kinetic energy is given by $E_{\text {kin. }}\left(R_{i j}\right)=R_{i j}^{2} / 4$ [see the discussion above Eq. (20)]. For the critical condition $\eta / 2=E_{\text {kin }}\left(R_{i j}\right)$, Eq. (13) becomes

$$
U\left(R_{i j}\right) \sim \frac{\beta}{\pi} R_{i j}
$$

As the potential barrier $\eta$ continues to increase beyond the critical value $R_{i j}^{2} / 2$, the RWA interaction (14) should remain unchanged since the physics in the rest frame does not change anymore. In the limit of $\eta \rightarrow \infty$, the interaction potential becomes the hard-core potential, i.e., $V\left(x_{i}-x_{j}\right)=\infty$ for $\left|x_{i}-x_{j}\right|<\beta$ and $V\left(x_{i}-x_{j}\right)=0$ for $\left|x_{i}-x_{j}\right|>\beta$. Therefore, the formula (14) is the RWA interaction for hard-core interaction.

\section{Coulomb interaction potential}

We now consider the case of Coulomb interaction potential, which is the dominant interaction for trapped ions [50-53]. We approach the Coulomb potential by using the following function:

$$
V\left(x_{i}-x_{j}\right)=\frac{\beta}{\sqrt{\left(x_{i}-x_{j}\right)^{2}+\varepsilon}} \quad \text { with } \varepsilon>0 .
$$

Obviously, in the limit of $\varepsilon \rightarrow 0$, the interaction $V\left(x_{i}-x_{j}\right)$ becomes the Coulomb potential. Applying formula (4) or (5), we obtain (more details provided in Appendix C)

$$
U\left(R_{i j}\right)=\frac{2 \beta^{*}}{\pi} \frac{1}{\sqrt{R_{i j}^{2}+\varepsilon}} .
$$

Here, we introduced the effective coupling

$$
\beta^{*} \equiv \beta K\left(\sqrt{\frac{R_{i j}^{2}}{R_{i j}^{2}+\varepsilon}}\right)
$$

where $K(x)$ is the complete elliptic integral of the first kind. Using the asymptotic approximation for the first complete elliptic integral [54], i.e., $K(i x) \approx \frac{1}{x} \ln (4 x)$ for $x \gg 1$. Eq. (15) has the following long-distance asymptotic behavior:

$$
U\left(R_{i j}\right) \approx \frac{2 \beta}{\pi R_{i j}} \ln \left(\frac{4 R_{i j}}{\sqrt{\varepsilon}}\right), \text { for } R_{i j} \gg 1 .
$$

We see that the RWA interaction (16) diverges in the limit of $\varepsilon \rightarrow 0$, which means the RWA interaction given by Eq. (4) and Eq. (5) is not valid for the Coulomb interaction potential. We will analyze the origin of this divergence and introduce the renormalization procedure to cancel the divergence.

\section{B. Renormalization procedure}

To find the origin of the divergence, we calculate the RWA interaction for Coulomb interaction potential using Eq. (5) by introducing a small cutoff $\tau_{c}$ as follows:

$U\left(R_{i j}\right)=\frac{2 \beta}{\pi R_{i j}} \int_{\tau_{c} \rightarrow 0}^{\pi / 2} \frac{1}{\sin \tau} d \tau=\left.\frac{2 \beta}{\pi R_{i j}} \ln \left(\frac{2}{\tau_{c}}\right)\right|_{\tau_{c} \rightarrow 0}$.

We see that $U\left(R_{i j}\right)$ diverges in the limit of $\tau_{c} \rightarrow 0$. Thus the divergence comes from the integral contribution inside the small interval $\left[0, \tau_{c}\right]$. For a given potential $V(r)$ with $r=$ $\left|x_{i}-x_{j}\right|$, we can estimate the integral inside the interval $\left[0, \tau_{c}\right]$ by

$$
U^{\tau_{c}} \equiv \frac{2}{\pi} \int_{0}^{\tau_{c}} V\left(R_{i j} \sin \tau\right) d \tau \approx \frac{2}{\pi} \int_{0}^{\tau_{c}} V\left(R_{i j} \tau\right) d \tau .
$$

If $U^{\tau_{c}}$ is finite, the potential $V(r)$ is well behaved inside the small distance $r<\tau_{c} R_{i j}$. However, for the Coulomb potential $V(r) \propto 1 / r, U^{\tau_{c}}$ is divergent. To obtain a finite meaningful $U\left(R_{i j}\right)$, we subtract this divergence by hand and get the following renormalized RWA interaction:

$$
\tilde{U}\left(R_{i j}\right) \equiv U\left(R_{i j}\right)-U^{\tau_{c}}=\frac{2}{\pi} \int_{\tau_{c}}^{\frac{\pi}{2}} V\left(R_{i j} \sin \tau\right) d \tau .
$$

Equivalently, we introduce the small cutoff $\tau_{c}$ to remove the divergence.

The detailed behavior of interaction potential $V(r)$ during the collision process is crucial. In the real physical process, if $V(r) \rightarrow \infty$ when $r \rightarrow 0$, the two particles can never touch each other since the two particles cannot overcome the potential barrier. The smallest distance $r_{c}$ they can approach during collision depends on their relative kinetic energy. If the relative kinetic energy of two particles is much larger than their interaction energy, the collision distance is very short, i.e., $r_{c} \ll 1$. When the two particles are far away, the interaction energy can be neglected and their kinetic energy in the centerof-mass frame is calculated: $E_{\mathrm{kin}}\left(R_{i j}\right)=\frac{1}{2}\left(\frac{R_{i j}}{2}\right)^{2}+\frac{1}{2}\left(\frac{R_{i j}}{2}\right)^{2}=$ $\frac{1}{4} R_{i j}^{2}$. Here, the kinetic energy of one particle with respect to the center of mass is given by $\frac{1}{2}\left(\frac{R_{i j}}{2}\right)^{2}$. We can calculate $r_{c}$ according to the energy-conservation law in the center-of-mass frame

$$
V\left(r_{c}\right)=E_{\mathrm{kin}}\left(R_{i j}\right)=\frac{1}{4} R_{i j}^{2}
$$

It is important to note here that $\tilde{U}\left(R_{i j}\right)$ is in fact a weighted time average of the interaction in the laboratory frame as revealed by Eq. (5). The result is that two particles, which collide during an oscillation period, already slow down as the 
they get closer. Therefore, we connect $\tau_{c}$ and $r_{c}$ by

$$
r_{c}=\gamma R_{i j} \sin \tau_{c} \approx \gamma R_{i j} \tau_{c},
$$

with the important collision factor $\gamma \gtrsim 1$, which is bigger than one, because of the extra interaction energy the particles accumulate as they move closer to each other and start to slow down. Therefore, we integrate to a radius that can be smaller than $r_{c}$. The collision factor $\gamma$ is phenomenologically introduced here, but we will calculate $\gamma$ for the interaction potentials discussed in this paper. Combining Eqs. (19), (20), and (21), we can obtain the explicit form of renormalized RWA interaction $\tilde{U}\left(R_{i j}\right)$.

For example, the collision $r_{c}$ of Coulomb interaction potential is calculated from Eq. (20),

$$
\frac{\beta}{r_{c}}=\frac{1}{4} R_{i j}^{2} \Rightarrow r_{c}=\frac{4 \beta}{R_{i j}^{2}} .
$$

Thus the cutoff $\tau_{c}$ is given by Eq. (21), i.e., $\tau_{c} \approx \frac{4 \beta}{\gamma R_{i j}^{3}}$. Therefore, the renormalized RWA interaction for Coulomb interaction is

$$
\tilde{U}\left(R_{i j}\right)=\frac{2 \beta}{\pi R_{i j}} \ln \left(2 / \tau_{c}\right) \equiv \frac{2 \beta^{*}}{\pi R_{i j}} .
$$

Here $\beta^{*} \equiv \beta \ln \left(\beta^{-1} \gamma R_{i j}^{3} / 2\right)$ is the renormalized coupling strength. The collision factor for Coulomb potential is $\gamma=e^{2}$, which is to be calculated in the next section.

\section{Inverse power-law interaction potential}

We now discuss a more general interaction potential form, i.e., the inverse power-law interaction potential as plotted in Fig. 2(a):

$$
V\left(x_{i}-x_{j}\right)=\frac{\beta^{2 n}}{\left|x_{i}-x_{j}\right|^{2 n}} .
$$

We restrict ourselves to integers and half-integers $n \geqslant 1 / 2$. If $n=1 / 2$, the potential $V\left(x_{i}-x_{j}\right)$ has the form of the Coulomb potential. If $n \rightarrow \infty$, the potential $V\left(x_{i}-x_{j}\right)=$ $\beta^{2 n} /\left|x_{i}-x_{j}\right|^{2 n}$ becomes the hard-core potential with a radius $\beta$. By applying Eq. (5), we obtain the RWA interaction

$$
\begin{aligned}
U\left(R_{i j}\right)= & \frac{2 \beta}{\pi R_{i j}^{2 n}} \int_{\varepsilon \rightarrow 0}^{\pi / 2} \frac{1}{\sin ^{2 n} \tau} d \tau \\
= & \frac{2 \beta}{\pi R_{i j}^{2 n}} \frac{1}{1-2 n}\left[{ }_{2} F_{1}\left(\frac{1}{2}, \frac{1}{2}-n ; \frac{3}{2}-n ; 1\right)\right. \\
& \left.-\left.\varepsilon^{1-2 n}{ }_{2} F_{1}\left(\frac{1}{2}, \frac{1}{2}-n ; \frac{3}{2}-n ; \varepsilon^{2}\right)\right|_{\varepsilon \rightarrow 0}\right] .
\end{aligned}
$$

Due to the term $\varepsilon^{1-2 n}$, the above integral diverges in the limit of $\varepsilon \rightarrow 0$ for $n>1 / 2$. Below, we will renormalize $U\left(R_{i j}\right)$ for integers $n \geqslant 1$, half-integers $n \geqslant 3 / 2$, and $n=1 / 2$ (Coulomb potential), respectively.

\section{Integers $n \geqslant 1$}

For integers $n \geqslant 1$, we use the properties of special functions ${ }_{2} F_{1}\left(\frac{1}{2}, \frac{1}{2}-n ; \frac{3}{2}-n ; 1\right)=0$ and ${ }_{2} F_{1}\left(\frac{1}{2}, \frac{1}{2}-n ; \frac{3}{2}-\right.$ $n ; 0)=1$. The renormalized RWA interaction can be obtained
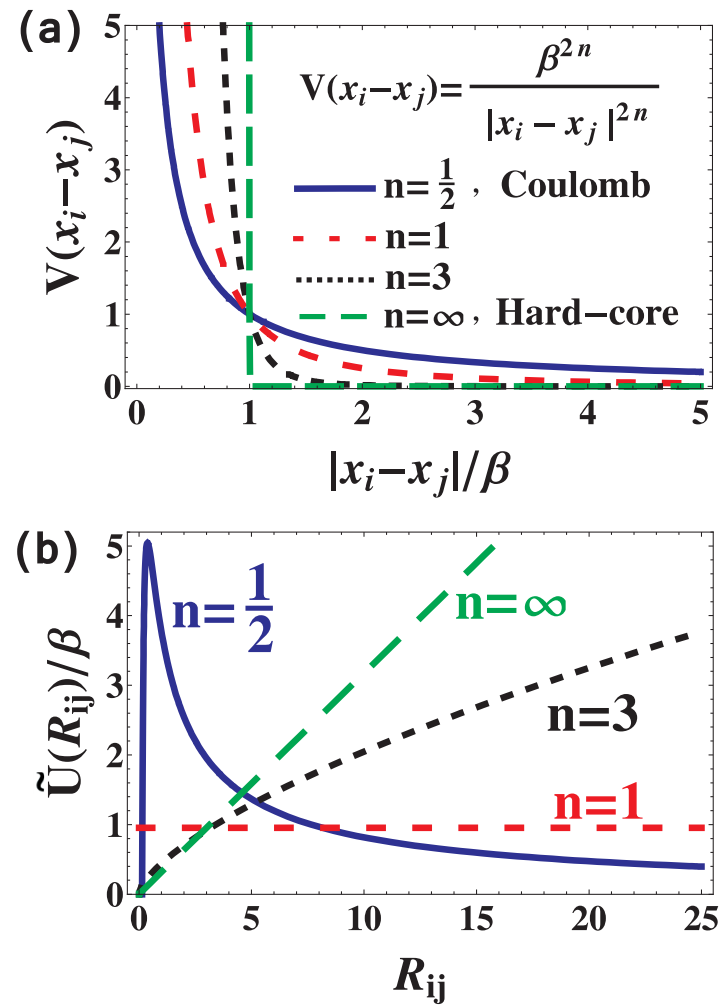

FIG. 2. Renormalized RWA interactions. (a) Inverse power-law interaction potentials and the example plots for $n=1 / 2$ (Coulomb interaction), $n=1, n=3$, and $n=\infty$ (hard-core interaction). (b) Renormalized RWA interaction potentials corresponding to the inverse power-law potentials shown in (a). Parameter: $\beta=0.01$.

from Eq. (25) by taking $\varepsilon=\tau_{c}$

$$
\tilde{U}\left(R_{i j}\right)=\frac{2 \beta^{2 n}}{\pi R_{i j}^{2 n}} \frac{1}{2 n-1} \tau_{c}^{1-2 n} .
$$

We use Eq. (20) to determine the collision distance $r_{c}$

$$
\frac{\beta^{2 n}}{r_{c}^{2 n}}=\frac{1}{4} R_{i j}^{2} \Rightarrow r_{c}=2^{\frac{1}{n}} \beta R_{i j}^{-\frac{1}{n}} .
$$

The truncation $\tau_{c}$ is given by $\tau_{c}=2^{\frac{1}{n}} \beta \gamma^{-1} R_{i j}^{-1-\frac{1}{n}}$. Plugging $\tau_{c}$ into Eq. (26), we get the explicit form of renormalized RWA interaction

$$
\tilde{U}\left(R_{i j}\right)=\frac{2 \beta \gamma^{2 n-1} 4^{\frac{1}{2 n}-1}}{\pi(2 n-1)} R_{i j}^{1-\frac{1}{n}} .
$$

The collision factor $\gamma$ will be determined later.

\section{Half-integers $n \geqslant 3 / 2$}

For half-integers $n=k+1 / 2$ with $k \geqslant 1$, we have the following divergence property of Eq. (25):

$$
\begin{aligned}
{ }_{2} & F_{1}\left(\frac{1}{2}, \frac{1}{2}-n ; \frac{3}{2}-n ; 1\right) \\
& =-\sqrt{\pi} \frac{\Gamma(k+1 / 2)}{\Gamma(k)} \frac{\sin (k \pi+\pi / 2)}{\cos (k \pi+\pi / 2)} \rightarrow \infty .
\end{aligned}
$$

The half-integers can be approached by taking $n=k+1 / 2+\epsilon$ with $\epsilon \rightarrow 0$. Thus we have the asymptotic behavior of the 
divergence above,

$$
{ }_{2} F_{1}\left(\frac{1}{2}, \frac{1}{2}-n ; \frac{3}{2}-n ; 1\right) \rightarrow \frac{1}{\epsilon} \frac{\Gamma(k+1 / 2)}{\sqrt{\pi} \Gamma(k)} .
$$

Different from the case of integers $n \geqslant 1$, where we have neglected the zero function ${ }_{2} F_{1}\left(\frac{1}{2}, \frac{1}{2}-n ; \frac{3}{2}-n ; 1\right)$ in Eq. (25), the divergence (29) appears in the function ${ }_{2} F_{1}\left(\frac{1}{2}, \frac{1}{2}-n ; \frac{3}{2}-\right.$ $n ; 1)$ for half-integers $n \geqslant 3 / 2$. It seems that the RWA interaction (28) is not valid for the case of half-integers $n \geqslant 3 / 2$. However, we show that this divergence is artificial and is canceled by another divergence in the function of ${ }_{2} F_{1}\left(\frac{1}{2}, \frac{1}{2}-n ; \frac{3}{2}-n ; \varepsilon^{2}\right)$. To reveal this, we write the function in Taylor's series [see identity (D4) in Appendix D]

$$
{ }_{2} F_{1}\left(\frac{1}{2}, \frac{1}{2}-n ; \frac{3}{2}-n ; \varepsilon^{2}\right)=\sum_{m=0}^{\infty} \frac{(1 / 2)_{m}(-k-\epsilon)_{m}}{k !(1-k-\epsilon)_{m}} \varepsilon^{2 m}
$$

The coefficient for $m=k$ in the limit of $\epsilon \rightarrow 0$ is

$$
\frac{(1 / 2)_{k}(-k-\epsilon)_{k}}{(1-k-\epsilon)_{k}} \frac{1}{k !}=\frac{1}{\epsilon} \frac{\Gamma(k+1 / 2)}{\sqrt{\pi} \Gamma(k)} .
$$

This coefficient is divergent as $\epsilon \rightarrow 0$ and cancels the divergence of (29). Therefore, the RWA interaction (28) is also valid for half-integers $n=k+1 / 2$ with $k \geqslant 1$.

\section{Coulomb potential $n=1 / 2$}

Assuming $n=1 / 2+\epsilon / 2$, the power-law interaction potential (24) becomes $V\left(x_{i}-x_{j}\right)=\beta\left|x_{i}-x_{j}\right|^{-1-\epsilon}$, which goes to the Coulomb interaction in the limit of $\epsilon \rightarrow 0$. Using the asymptotic property, ${ }_{2} F_{1}\left(\frac{1}{2}, \frac{1}{2}-n ; \frac{3}{2}-n ; 1\right) \approx 1-\epsilon \ln 2$ (see the proof in Appendix D), we have from Eq. (25)

$$
U\left(R_{i j}\right)=\left.\frac{2 \beta}{\pi R_{i j}} \ln \left(\frac{2}{\tau_{c}}\right)\right|_{\epsilon=0, \tau_{c} \ll 1} .
$$

Compared to formula (23), it is just the case of Coulomb potential (more details are provided in Appendix D).

\section{Collision factor $\gamma$}

Now we discuss how to determine the collision factor $\gamma$ for inverse power-law potential. In principle, the collision factor $\gamma$ is phenomenologically introduced in Eq. (21). Here, we determine it using the correspondence conditions. From the expression (24) we see that the inverse power-law potential approaches the hard-core potential in the limit of $n \rightarrow \infty$ :

$$
\frac{\beta^{2 n}}{\left(x_{i}-x_{j}\right)^{2 n}} \rightarrow \begin{cases}\infty & \text { if }\left|x_{i}-x_{j}\right|<\beta, \\ 0 & \text { if }\left|x_{i}-x_{j}\right|>\beta .\end{cases}
$$

Comparing the RWA interaction (28) to Eq. (14), we get the first correspondence condition

$$
\gamma^{2 n-1} /(2 n-1) \rightarrow 2 \text { for } n \rightarrow \infty \text {. }
$$

The simplest assumption is $\gamma^{2 n-1}=4 n+c$, where $c$ is a free parameter to be further determined. Thus the collision factor takes the form of $\gamma=(4 n+c)^{1 /(2 n-1)}$. This form needs to be valid for the Coulomb potential, i.e., $n=1 / 2$. By writing $n=1 / 2+\varepsilon / 4$ with $\varepsilon \rightarrow 0$, we have

$$
\gamma=(2+c+\varepsilon)^{2 / \varepsilon} \rightarrow(2+c)^{2 / \varepsilon} e^{2(2+c)} \text { for } \varepsilon \rightarrow 0 .
$$

The parameter $c$ can only take the value of -1 to get a meaningful result. Otherwise, the prefactor $(2+c)^{2 / \varepsilon} \rightarrow$ $(2+c)^{\infty}$ takes either zero or infinity, both of which are unphysical. Finally, we get the expression for the collision factor $\gamma$ for the inverse power-law interaction potential

$$
\gamma=(4 n-1)^{\frac{1}{2 n-1}} \text {. }
$$

The collision factor becomes $\gamma=e^{2}$ for Coulomb interaction $(n=1 / 2)$ and $\gamma=1$ for hard-core interaction $(n \rightarrow \infty)$.

\section{Summary}

We summarize our results of RWA interaction for the inverse power-law potential as follows:

$$
\tilde{U}\left(R_{i j}\right)= \begin{cases}\frac{2 \beta}{\pi R_{i j}} \ln \left(\beta^{-1} \gamma R_{i j}^{3} / 2\right) & \text { for } n=\frac{1}{2}, \\ \frac{2 \beta \gamma^{2 n-1} 4 \frac{1}{2 n}-1}{\pi(2 n-1)} R_{i j}^{1-\frac{1}{n}} & \text { for } n=1, \frac{3}{2}, 2, \frac{5}{2}, \ldots, \\ \frac{\beta}{\pi} R_{i j} & \text { for } n \rightarrow \infty .\end{cases}
$$

The collision factor is given by $\gamma=(4 n-1)^{\frac{1}{2 n-1}}$. We see that the renormalized RWA interaction for the Coulomb potential $(n=1 / 2)$ still keeps the form of Coulomb's law, up to logarithmic corrections. We show the behaviors of $\tilde{U}\left(R_{i j}\right)$ for several cases in Fig. 2(b). For every interaction potential with $n>1, \tilde{U}\left(R_{i j}\right)$ actually grows with $R_{i j}$. It is also interesting to note that for the case of $n=1$, the corresponding $\tilde{U}\left(R_{i j}\right)$ is a constant, which means there is no effective interaction in the slow dynamics of $X_{i}(t)$ and $P_{i}(t)$.

\section{Lennard-Jones interaction potential}

Another general choice to describe the interaction between two noble atoms or molecules is the Lennard-Jones interaction potential

$$
V\left(x_{i}-x_{j}\right)=4 \epsilon\left(\frac{\sigma^{2 m}}{\left|x_{i}-x_{j}\right|^{2 m}}-\frac{\sigma^{m}}{\left|x_{i}-x_{j}\right|^{m}}\right),
$$

where $\epsilon$ defines the interaction strength, $\sigma$ defines the interaction range, and the parameter $m$ is usually taken $m=6$ in the study. By introducing the cutoff $\tau_{c}$, we obtain the RWA interaction from Eq. (19)

$$
\tilde{U}\left(R_{i j}\right)=\frac{8 \epsilon}{\pi}\left(\frac{\sigma^{2 m}}{R_{i j}^{2 m}} \frac{1}{2 m-1} \tau_{c}^{1-2 m}-\frac{\sigma^{2 m}}{R_{i j}^{m}} \frac{1}{m-1} \tau_{c}^{1-m}\right) .
$$

Then from Eq. (20) and Eq. (21) we can calculate the cutoff as follows:

$$
\tau_{c}=\frac{\sigma}{\gamma R_{i j}}\left(\frac{1}{2}+\frac{1}{2} \sqrt{1+\frac{1}{4 \epsilon} R_{i j}^{2}}\right)^{-1 / m} .
$$

As seen from Eq. (36), the Lennard-Jones potential is composed of two inverse power-law potentials with exponents $2 m$ and $m$, respectively. During the collision in the range of small distance $\left|x_{i}-x_{j}\right|<\sigma$, we have $\frac{\sigma^{2 m}}{\left|x_{i}-x_{j}\right|^{2 m}} \gg \frac{\sigma^{m}}{\left|x_{i}-x_{j}\right|^{m}}$. Therefore, the term $\frac{\sigma^{2 m}}{\left|x_{i}-x_{j}\right|^{2 m}}$ in Eq. (36) is dominant during the collision. The collision factor of the Lennard-Jones potential can be calculated from Eq. (34) by choosing $n=m$, i.e., $\gamma=(4 m-1)^{\frac{1}{2 m-1}}$. 
(a)

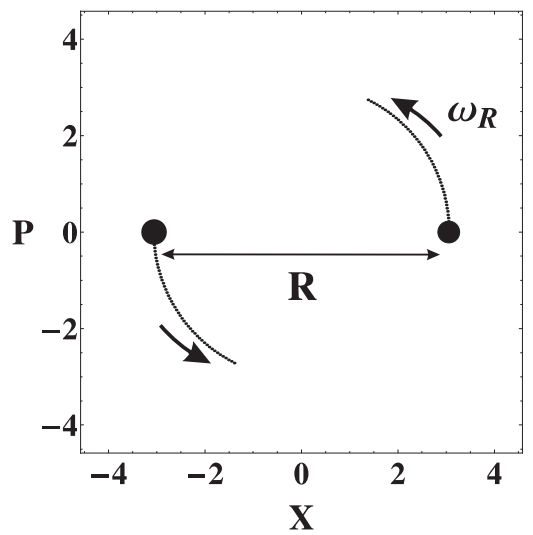

(b)

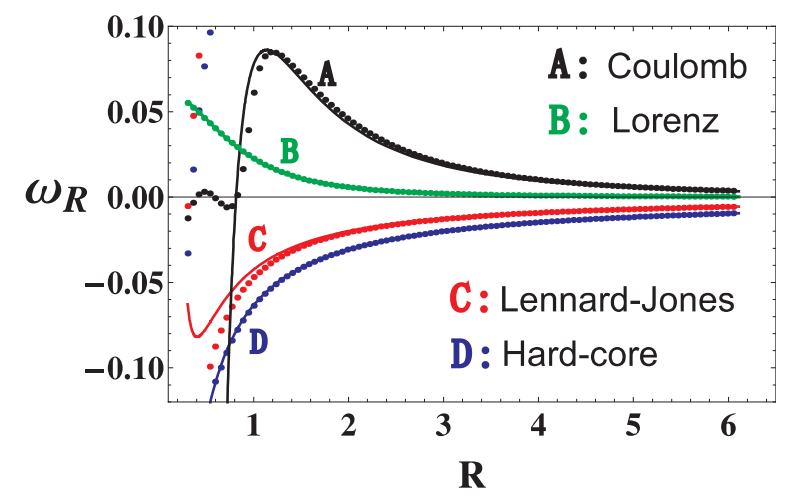

FIG. 3. Two-body dynamics for different interactions. (a) In the presence of interaction, the two particles start to rotate at a frequency $\omega_{R}$, which depends on their phase-space distance $R$. (b) Rotating frequency $\omega_{R}$ as function of $R$. The dots represent the data from Poincaré mapping, while the solid lines are given by Eq. (39). Different colors represent different interactions as indicated on the plot. Interaction potential parameters: $\beta=0.1, \varepsilon=1$ for Lorenz interaction; $\beta=0.1$ for Coulomb interaction; $\epsilon=0.01, \sigma=0.1$ for Lennard-Jones interaction; hard-core interaction is modeled by the inverse power-law interaction with $\beta=0.1, n=20$.

\section{MANY-BODY DYNAMICS}

\section{A. Two-body dynamics}

To justify our RWA interaction, we consider the two-body dynamics with resonant condition $\delta=0$ and zero driving limit $\Lambda \rightarrow 0$. In Fig. 3(a), we show the trajectories of two particles in phase space with symmetric initial conditions, i.e., $\left(X_{1}(0)=\right.$ $\left.\frac{1}{2} R, P_{1}(0)=0\right)$ and $\left(X_{2}(0)=-\frac{1}{2} R, P_{2}(0)=\pi\right)$. Without interaction, both particles are performing independent harmonic oscillations with time period $\Delta t=2 \pi$. Therefore, the positions of two particles in phase space are fixed points as shown by two black dots in Fig. 3(a). In the presence of interaction, the two particles start to rotate with a frequency $\omega_{R}$ as shown in the same figure. The rotating frequency $\omega_{R}$ depends on phase-space distance $R$ of the two particles. In this simple case, the motions of the two particles are symmetric. The dynamics of either particle, e.g., the first particle, is given by $X_{1}(t)=\frac{1}{2} R \cos \left(\omega_{R} t\right), P_{1}(t)=\frac{1}{2} R \sin \left(\omega_{R} t\right)$. The rotating frequency $\omega_{R}$ can be determined from the $\operatorname{EOM}(9)$,

$$
\omega_{R}=-\frac{2}{R} \frac{d \tilde{U}(R)}{d R} .
$$

Here, $\tilde{U}(R)$ is the renormalized RWA interaction.
The two-body rotation frequency $\omega_{R}$ can also be obtained from Poincaré mapping. We simulate the dynamics of two particles $\left(x_{i}(t), p_{i}(t)\right)$ based on the EOM (6) and take their values stroboscopically every time period $\Delta t=2 \pi$. This gives the trajectories of two particles in phase space as shown by the two dotted lines in Fig. 3(a). In Fig. 3(b), we compared the numerically extracted $\omega_{R}$ to the analytical expression (39) for different types of interactions, i.e., Lorenz interaction, Coulomb interaction, hard-core interaction, and Lennard-Jones interaction. We see that the result (39) is very good for large $R$ and breaks down for small $R$. From Eq. (21), we see the relative kinetic energy of two particles is $R^{2} / 4$. Therefore, we conclude that the frequency $\omega_{R}$ as function of $R$ given by Eq. (39) is valid when the interaction energy is much smaller than the relative kinetic energy of two particles, i.e., the cutoff given by Eq. (21) satisfies $r_{c} \ll 1$.

\section{B. Three-body dynamics}

As we discussed above, while the two-body dynamics can be solved analytically, the three-body dynamics cannot be obtained analytically from the RWA EOM (9) in general. Thus we solve the three-body problem via numerical simulation. In Fig. 4, we compare the trajectories based on the original EOM (6) with the trajectories given by RWA EOM (9). The results for the Coulomb, hard-core, and Lennard-Jones interactions are given in the left, middle, and right columns of Fig. 4, respectively. In the upper figure of each column, the colored dots (blue, green, and red) represent the three particles' initial positions in phase space. The colored solid lines are the trajectories obtained from RWA EOM (9). The black dotted lines are the results from the original EOM (6) combined with Poincaré mapping. In the lower figure of each column, we show the time evolution of $r_{i}(t)=\sqrt{X_{i}^{2}(t)+P_{i}^{2}(t)}, i=1,2,3$ obtained from RWA EOM (9) (solid lines) and the Poincaré mapping (dotted lines).

We see that the agreement is good. The discrepancy in the long-time limit comes from the rotating wave approximation we used in this paper. Depending on the type of interactions and the initial conditions, the three interacting particles may have complex trajectories in phase space. In the figures for Coulomb interaction, particle 2 and particle 3 can be viewed as a two-body subsystem while their center of mass forms another larger two-body system with particle 1. The RWA interaction corresponding to Coulomb interaction decays with phase-space distance. Thus, as shown in the lower figure of this column, the rotational frequency of particle 1 around the center of particle 2 and 3 is much slower than the rotating frequency of particle 2 and 3 around each other. For the figures for the hard-core interaction, the corresponding RWA interaction increases linearly with the phase-space distance. Therefore, the interaction between particle 1 and particle 2 or between particle 1 and particle 3 is stronger than interaction between particle 2 and particle 3 . As a result, the orbits of particle 2 and particle 3 are not as regular as in the case of Coulomb interaction due to the strong disturbance by particle 1 . For the Lennard-Jones interaction, we change the initial conditions of the three particles. From the lower figure, we see that the three particles exchange their positions in phase space with a period of about $240 \Delta t$ as indicated by the arrows. The parameters for the interactions are given in the caption of Fig. 4. 
Coulomb
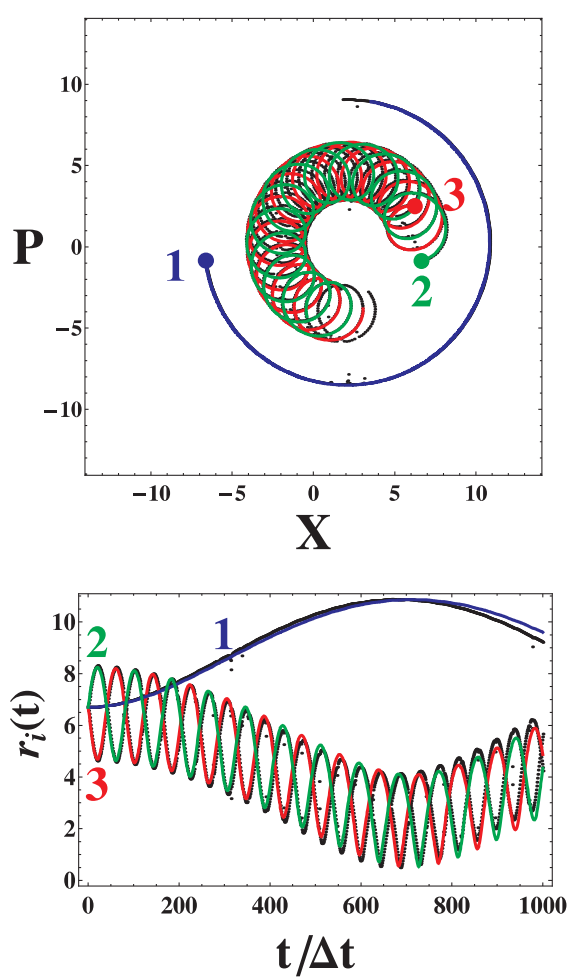

Hard-core
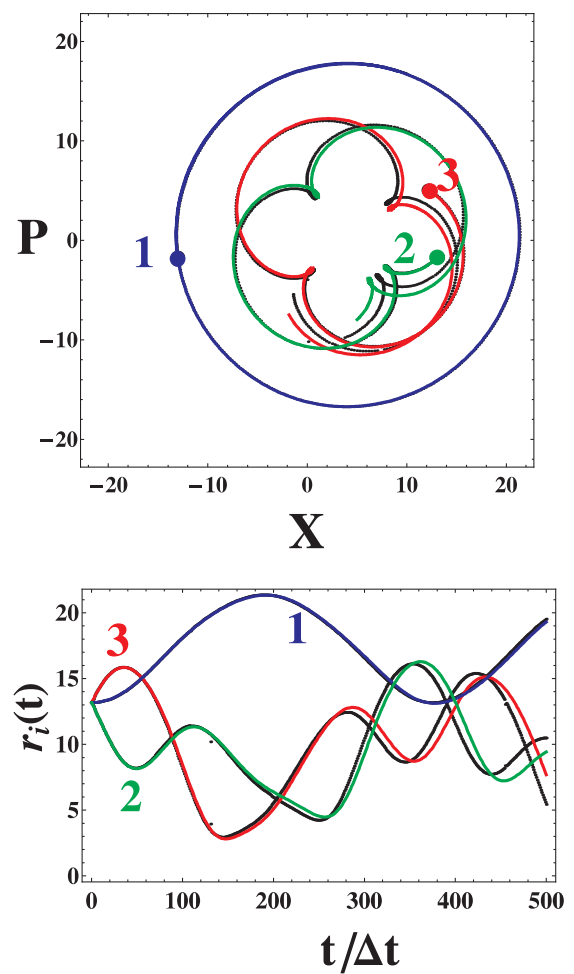

Lennard-Jones
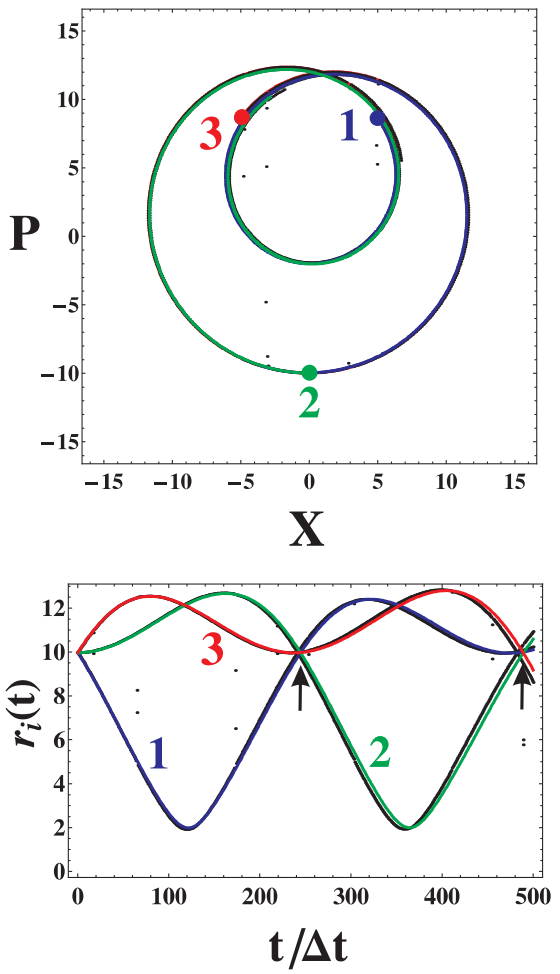

FIG. 4. Three-body dynamics for different interactions. (Left column) The upper figure shows the trajectories of three particles in the presence of Coulomb interaction with $\beta=0.1$. The colored dots represent the initial conditions and the colored solid lines represent the dynamics from RWA EOM (9). The black dotted lines are the data from EOM (6) combined with Poincaré mapping. The lower figure shows the time evolution of $r_{i}(t)=\sqrt{X_{i}^{2}(t)+P_{i}^{2}(t)}, i=1,2,3$ obtained from RWA EOM (9) (solid colored lines) and the Poincaré mapping (dotted black lines). (Middle column) The middle two figures show the three-body dynamics for hard-core interaction with $\beta=0.1$. (Right column) The right two figures show the three-body dynamics for Lennard-Jones interaction with parameters $\epsilon=0.01$ and $\sigma=0.1$.

\section{Dynamics under driving}

In the above discussion, we justify the RWA interaction without consideration of driving field. Now we add the driving term to the EOM and justify the RWA interaction. In Fig. 5 we show the two-body dynamics under driving. We choose the driving strength $\Lambda=0.1$ in the RWA Hamiltonian (3). (a)

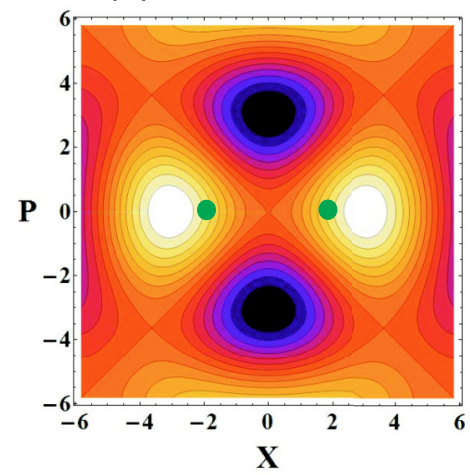

(b)

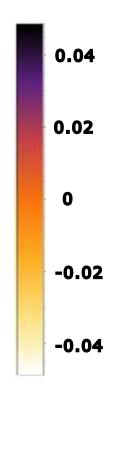

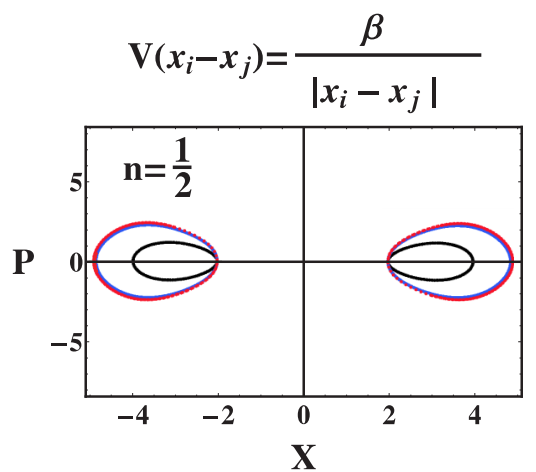

(c)

$$
\mathrm{V}\left(x_{i}-x_{j}\right)=\frac{\beta^{2}}{\left|x_{i}-x_{j}\right|^{2}}
$$

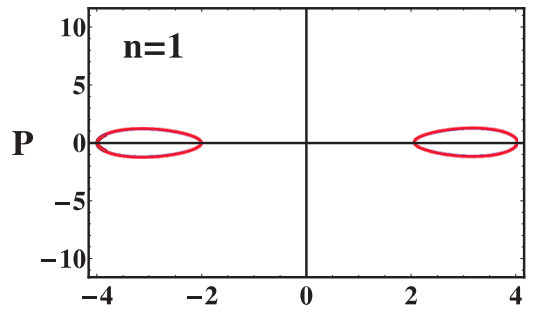

FIG. 5. Two-body dynamics under driving. (a) Contour plot of single-particle Hamiltonian function (40) in phase space and the initial conditions of two particles (green dots). (b) The trajectories of two particles in phase space without interaction (black, $\beta=0$ ) and with Coulomb interaction (red and blue, $\beta=0.1$ ). The black and red trajectories are obtained from the time evolution based on the original EOM (6) combined with the technique of Poincaré mapping. The blue trajectories are obtained from the time evolution based the RWA EOM (9). Driving parameter: $\Lambda=0.1$. (c) The trajectories of two particles in phase space with interaction potential $V\left(x_{i}-x_{j}\right)=\beta^{2} /\left(x_{i}-x_{j}\right)^{2}$. All the trajectories with interaction (red and blue, $\beta=0.1$ ) and without interaction (black, $\beta=0$ ) completely overlap each other. Driving parameter: $\Lambda=0.1$. 
(a)

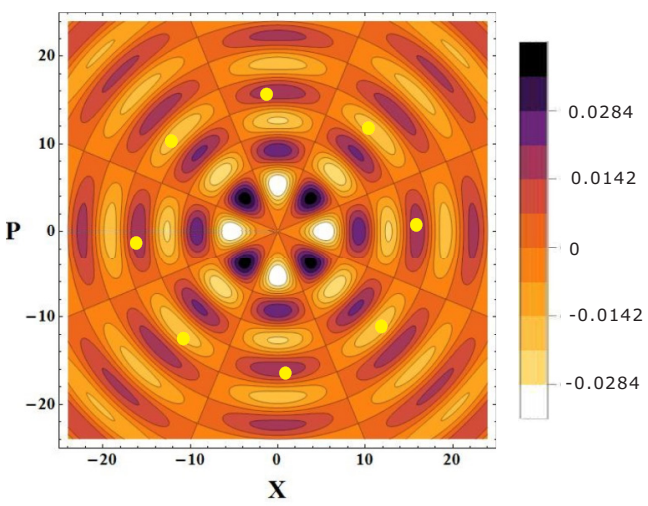

(b)

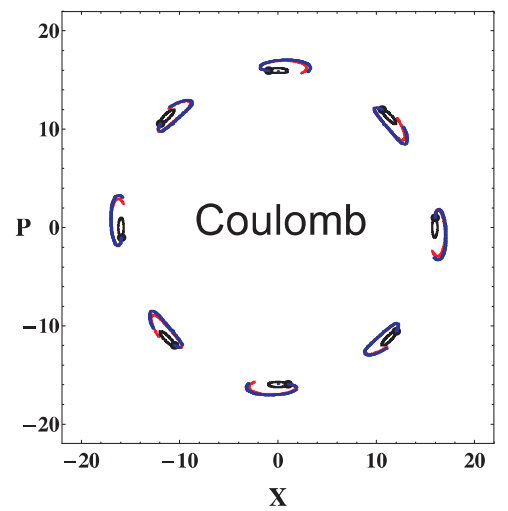

(c)

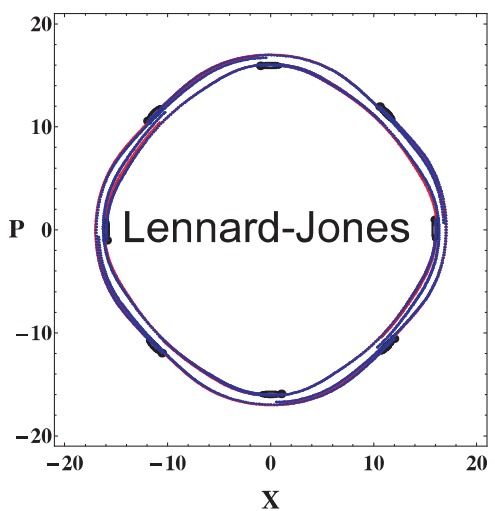

FIG. 6. Eight-body dynamics under driving. (a) Contour plot of single-particle Hamiltonian function (40) in phase space with $\delta=0$, $\Lambda=0.1$, and $k=4$. The yellow dots indicate the initial conditions of eight particles. (b) The trajectories of eight particles in phase space without interaction (black) and with Coulomb interaction (red and blue, $\beta=0.1$ ). Driving parameter: $\Lambda=0.1$. (c) The trajectories of eight particles in phase space with Lennard-Jones interaction (red and blue, $\sigma=0.1$ and $\epsilon=0.01$ ). Driving parameter: $\Lambda=0.1$.

We further consider the special resonant condition that the driving frequency is twice of the harmonic frequency in the RWA Hamiltonian (3), i.e., $g=g_{1}+g_{2}+U\left(R_{12}\right)$. Here, $g_{i}$ with $i=1,2$ representing the index of two particles is defined as the single-particle Hamiltonian function

$$
g_{i}=\frac{1}{2} \delta r_{i}^{2}+\Lambda \cos \left(\frac{k}{2} \pi\right) J_{k}\left(r_{i}\right) \cos \left(k \theta_{i}\right) .
$$

In Fig. 5(a) we plot the single-particle Hamiltonian function in phase space with chosen parameters $\delta=0$ and $k=2$, i.e., $g_{i}=$ $-\Lambda J_{2}\left(r_{i}\right) \cos \left(2 \theta_{i}\right)$. Due to the driving field, the system hosts multiple stable vibrational states with different amplitudes and phases. We only draw the four lowest vibrational stable states with different phases (centers of the bright and dark regions). Now we put two particles (two green dots) near two stable states with phase 0 and $\pi$, respectively, and study their motions in the presence of interaction. In Figs. 5(b) and 5(c), we compare the full numerical simulations to the RWA dynamcis. The trajectories with black and red colors are the results from the time evolution based on the original EOM (6) with consideration of the two particles' interaction $V\left(x_{i}-x_{j}\right)$ combined with the technique of Poincaré mapping. The trajectories with blue color are the results from the time evolution based on the RWA EOM (9) with the renormalized $\tilde{U}\left(R_{i j}\right)$. We see that the results agree with each other. Especially we find, for the case of $n=1$, the trajectories for $\beta=0$ and $\beta \neq 0$ overlap completely as shown in Fig. 5(c). This means the inverse power-law potential for $n=1$ generates no interaction under RWA.

Figure 6 shows the dynamics of eight interacting particles. In Fig. 6(a) we plot the single-particle Hamiltonian function (40) with parameters $\delta=0, \Lambda=0.1$, and $k=4$. The initial conditions are represented by the eight yellow dots. In Fig. 6(b) and Fig. 6(c) we show the trajectories of eight particles under driving. Without interaction the eight particles exhibit localized motions as illustrated by the black curves. In the case of Coulomb interaction, the trajectories of eight particles are enlarged a bit as shown in Fig. 6(b) by the red orbits (from Poincaré mapping) and blue orbits [from RWA EOM (9)]. The RWA interaction corresponding to Coulomb interactions de- cays with phase-space distance, which can be viewed as small perturbation when the particles are far away in phase space. In the case of the Lennard-Jones interaction, the trajectories of eight particles become global motions as shown in Fig. 6(c). This reflects the fact that the RWA interaction corresponding to the Lennard-Jones interaction increases as the phase-space distance increases, which is similar to the hard-core interaction.

\section{SUMMARY AND OUTLOOK}

We investigated the classical dynamics of periodically driven interacting particles in a 1D harmonic trap. Under RWA, we transform the real-space interaction $V\left(x_{i}-x_{j}\right)$ into a RWA interaction in phase space. Particularly, we find an effective long-distance RWA interaction can be produced by short-distance real-space interactions, e.g., pointlike $\delta$ function interaction and hard-core interaction. The RWA interaction describes the effect of the interaction on the slowly changing amplitude and phase of the globally oscillating particles, while the fast oscillations take on the role of a force carrier, which allows for interaction over much larger effective distances.

We solved the divergence problem by introducing the renormalization procedure. For the Coulomb interaction, our renormalization procedure just eliminates the high energy collision process, which gives rise to a renormalized strength of Coulomb interaction. For the hard-core interaction, our renormalization procedure gives rise to a completely different longdistance interaction, which increases linearly with phase-space distance. We justified our theory by simulating the many-body dynamics numerically in the presence of various interaction potentials like pointlike $\delta$ interaction, Coulomb interaction, hard-core interaction, and Lennard-Jones interaction.

The work in this paper only focuses on the classical dynamics of interacting 1D particles. The next step is to extend our study to the quantum regime. An interesting direction is combing the lattice structure created by the driving [23], as shown in Fig. 6(a), together with the effective RWA interaction. In this way, a Hubbard model can be simulated in phase space. This will provide another way to study strongly correlated systems by periodically driving $1 \mathrm{D}$ system. 


\section{ACKNOWLEDGMENTS}

L.G. and M.L. acknowledge support from the KHYSGaststipendium (Visiting Researcher Scholarship from Karlsruhe House of Young Scientist, Karlsruher Institut für Technologie). L.G. also acknowledges financial support from Carl-Zeiss-Stiftung (0563-2.8/508/2).

\section{APPENDIX A: DERIVATION OF RWA INTERACTION}

The Hamiltonian of many trapped interacting particles under periodic driving can be written as

$$
\begin{aligned}
H(t)= & \sum_{i}\left[\frac{p_{i}^{2}}{2}+\frac{x_{i}^{2}}{2}+\Lambda \cos (\Omega t) \cos \left(x_{i}\right)\right] \\
& +\sum_{i<j} V\left(x_{i}-x_{j}\right) .
\end{aligned}
$$

Here, all the quantities are scaled to be dimensionless. We are working in the regime near the resonant condition, i.e., $\Omega \approx k$ with a positive integer $k$. Using the generating function of the second kind

$$
\begin{aligned}
G_{2}(\vec{x}, \vec{P}, t)= & \sum_{i} \frac{x_{i} P_{i}}{\cos (\Omega t / k)}-\frac{1}{2} x_{i}^{2} \tan \left(\frac{\Omega}{k} t\right) \\
& -\frac{1}{2} P_{i}^{2} \tan \left(\frac{\Omega}{k} t\right),
\end{aligned}
$$

we transform to the rotating frame with frequency $\Omega / k$. Here, $\vec{x}=\left(x_{1}, x_{2}, \ldots\right)$ and $\vec{P}=\left(P_{1}, P_{2}, \ldots\right)$ represent the assembled canonical coordinates of all the particles. The corresponding canonical transformations of coordinates and momenta are

$$
p_{i}=\frac{\partial G_{2}(\vec{x}, \vec{P}, t)}{\partial x_{i}}, \quad X_{i}=\frac{\partial G_{2}(\vec{x}, \vec{P}, t)}{\partial P_{i}},
$$

which results in

$$
\begin{aligned}
& x_{i}=P_{i} \sin \left(\frac{\Omega}{k} t\right)+X_{i} \cos \left(\frac{\Omega}{k} t\right), \\
& p_{i}=P_{i} \cos \left(\frac{\Omega}{k} t\right)-X_{i} \sin \left(\frac{\Omega}{k} t\right) .
\end{aligned}
$$

The canonical transformation of $H(t)$ itself is

$$
\begin{aligned}
K(t) \equiv & H(t)+\partial G_{2} / \partial t=\sum_{i} \frac{1}{2} \delta\left(X_{i}^{2}+P_{i}^{2}\right) \\
& +\Lambda \cos (\Omega t) \cos \left[P_{i} \sin (\Omega t / k)+X_{i} \cos (\Omega t / k)\right] \\
& +\sum_{i<j} V\left[\Delta P_{i, j} \sin (\Omega t / k)+\Delta X_{i, j} \cos (\Omega t / k)\right] \\
= & \sum_{i} \frac{1}{2} \delta\left(X_{i}^{2}+P_{i}^{2}\right) \\
& +\frac{\Lambda}{2}\left[\cos (\Omega t) e^{i\left[P_{i} \sin (\Omega t / k)+X_{i} \cos (\Omega t / k)\right]}+\text { H.c. }\right] \\
& +\sum_{i<j} V\left[\Delta P_{i, j} \sin (\Omega t / k)+\Delta X_{i, j} \cos (\Omega t / k)\right] .
\end{aligned}
$$

Here, we have defined the detuning $\delta \equiv 1-\Omega / k$, the displacement of one particle in phase space $r_{i} e^{i \theta_{i}} \equiv X_{i}+i P_{i}$, and the relative displacement of two particles in phase space $\left(\Delta X_{i j}, \Delta P_{i j}\right) \equiv\left(X_{i}-X_{j}, P_{i}-P_{j}\right)$. Using the Jacobi-Anger expansion,

$$
e^{i z \cos \varphi}=\sum_{-\infty}^{+\infty} i^{m} J_{m}(z) e^{i m \varphi},
$$

we have

$$
\begin{aligned}
& \cos (\Omega t) e^{i\left[P_{i} \sin (\Omega t / k)+X_{i} \cos (\Omega t / k)\right]} \\
& \quad=\cos (\Omega t) e^{i r_{i} \cos \left(\Omega t / k-\theta_{i}\right)} \\
& =\cos (\Omega t) \sum_{-\infty}^{+\infty} i^{m} J_{m}\left(r_{i}\right) e^{-i m \theta_{i}} e^{i m \Omega t / k} \\
& =\frac{1}{2}\left(e^{i \Omega t}+e^{-i \Omega t}\right) \sum_{-\infty}^{+\infty} i^{m} J_{m}\left(r_{i}\right) e^{-i m \theta_{i}} e^{i m \Omega t / k} .
\end{aligned}
$$

Under rotating wave approximation (RWA), we drop fast oscillating terms in Eq. (A6) and obtain

$$
\begin{aligned}
& \cos (\Omega t) e^{i\left[P_{i} \sin (\Omega t / k)+X_{i} \cos (\Omega t / k)\right]} \\
& \quad \rightarrow \frac{1}{2}\left[i^{k} J_{k}\left(r_{i}\right) e^{-i k \theta_{i}}+i^{-k} J_{-k}\left(r_{i}\right) e^{i k \theta_{i}}\right] \\
& \quad=e^{i k \frac{\pi}{2}} J_{k}\left(r_{i}\right) \cos \left(k \theta_{i}\right) .
\end{aligned}
$$

Now we focus on the interacting term in Eq. (A5)

$$
\begin{aligned}
V & {\left[\Delta P_{i, j} \sin (\Omega t / k)+\Delta X_{i, j} \cos (\Omega t / k)\right] } \\
& =V\left[R_{i, j} \cos \left(\Omega t / k-\theta_{i, j}\right)\right] \\
& =\int_{-\infty}^{+\infty} d q V_{q} e^{i q R_{i, j} \cos \left(\Omega t / k-\theta_{i, j}\right)} \\
& =\sum_{m=-\infty}^{+\infty} i^{m} \int_{-\infty}^{+\infty} d q V_{q} J_{m}\left(q R_{i j}\right) e^{i m\left(\Omega t / k-\theta_{i, j}\right)} .
\end{aligned}
$$

Here, $R_{i j} e^{i \theta_{i j}} \equiv\left(\Delta X_{i j}, \Delta P_{i j}\right)$, and $V_{q}$ is the coefficient of Fourier transformation of potential, i.e.,

$$
V_{q}=\frac{1}{2 \pi} \int_{-\infty}^{+\infty} d x V(x) e^{-i q x} .
$$

In the RWA, we drop all the terms with $m \neq 0$ in Eq. (A8) and obtain the RWA interaction potential in phase space

$$
U\left(R_{i j}\right)=\int_{-\infty}^{+\infty} d q V_{q} J_{0}\left(q R_{i j}\right) .
$$

We then apply the integral representation of Bessel function, i.e.,

$$
J_{0}(x)=\frac{1}{2 \pi} \int_{-\pi}^{+\pi} e^{-i x \sin \tau} d \tau,
$$

and get an alternative form of Eq. (A9)

$$
\begin{aligned}
U\left(R_{i j}\right) & =\int_{-\infty}^{+\infty} d q V_{q} J_{0}\left(q R_{i j}\right) \\
& =\int_{-\infty}^{+\infty} d q V_{q} \frac{1}{2 \pi} \int_{-\pi}^{+\pi} e^{-i q R_{i j} \sin \tau} d \tau \\
& =\frac{1}{2 \pi} \int_{-\pi}^{+\pi} V\left(R_{i j} \sin \tau\right) d \tau .
\end{aligned}
$$


Normally, the potential is only a function of the distance $\left|x_{i}-x_{j}\right|$ which implies $V(x)=V(-x)$. Thus we have

$$
\begin{aligned}
U\left(R_{i j}\right) & =\frac{1}{2 \pi} \int_{-\pi}^{+\pi} V\left(R_{i j} \sin \tau\right) d \tau \\
& =\frac{2}{\pi} \int_{0}^{\frac{\pi}{2}} V\left(R_{i j} \sin \tau\right) d \tau .
\end{aligned}
$$

Finally, we get the RWA Hamiltonian of all interacting particles in phase space

$$
g=\sum_{i}\left[\frac{1}{2} \delta r_{i}^{2}+\Lambda \cos \left(\frac{k \pi}{2}\right) J_{k}\left(r_{i}\right) \cos \left(k \theta_{i}\right)\right]+\sum_{i<j} U\left(R_{i j}\right),
$$

where $R_{i j}=\left|r_{i} e^{i \theta_{i}}-r_{j} e^{i \theta_{j}}\right|=\sqrt{\left(X_{i}-X_{j}\right)^{2}+\left(P_{i}-P_{j}\right)^{2}}$ is the phase-space distance.

\section{APPENDIX B: CANONICAL EQUATIONS OF MOTION IN THE ROTATING FRAME}

The canonical equations of motion given by RWA Hamiltonian (A12) in the rotating frame are

$$
\frac{d X_{i}}{d t}=\frac{\partial g}{\partial P_{i}}, \quad \frac{d P_{i}}{d t}=-\frac{\partial g}{\partial X_{i}} .
$$

From the relationship

$$
X_{i}=r_{i} \cos \theta_{i}, \quad P_{i}=r_{i} \sin \theta_{i}, \quad r_{i}=\sqrt{X_{i}^{2}+P_{i}^{2}},
$$

we have

$$
\begin{aligned}
\frac{\partial \theta_{i}}{\partial X_{i}} & =\frac{-P_{i}}{X_{i}^{2}+P_{i}^{2}}, \quad \frac{\partial \theta_{i}}{\partial P_{i}}=\frac{X_{i}}{X_{i}^{2}+P_{i}^{2}}, \\
\frac{\partial r_{i}}{\partial X_{i}} & =\frac{X_{i}}{\sqrt{X_{i}^{2}+P_{i}^{2}}}, \quad \frac{\partial r_{i}}{\partial P_{i}}=\frac{P_{i}}{\sqrt{X_{i}^{2}+P_{i}^{2}}} .
\end{aligned}
$$

As a result, the explicit form of canonical equations of motion are

$$
\begin{aligned}
\frac{d X_{i}}{d t}= & \delta P_{i}+\Lambda \cos \left(\frac{k \pi}{2}\right) \\
& \times\left[J_{k}^{\prime}\left(r_{i}\right) \cos \left(n \theta_{i}\right) \frac{\partial r_{i}}{\partial P_{i}}-k J_{k}\left(r_{i}\right) \sin \left(k \theta_{i}\right) \frac{\partial \theta_{i}}{\partial P_{i}}\right] \\
& +\sum_{j} U^{\prime}\left(R_{i j}\right) \frac{P_{i}-P_{j}}{R_{i j}}, \\
\frac{d P_{i}}{d t}= & -\delta X_{i}-\Lambda \cos \left(\frac{k \pi}{2}\right) \\
& \times\left[J_{k}^{\prime}\left(r_{i}\right) \cos \left(k \theta_{i}\right) \frac{\partial r_{i}}{\partial X_{i}}-k J_{k}\left(r_{i}\right) \sin \left(k \theta_{i}\right) \frac{\partial \theta_{i}}{\partial X_{i}}\right] \\
& -\sum_{j} U^{\prime}\left(R_{i j}\right) \frac{X_{i}-X_{j}}{R_{i j}} .
\end{aligned}
$$

Based on EOM (B4), we can calculate the trajectories of interacting particles in phase space.

\section{APPENDIX C: EXAMPLES OF INTERACTION POTENTIALS}

\section{Rectangular potential}

We define the following short-range interaction potential by a rectangular function:

$$
\eta \operatorname{rect}\left(\frac{x_{i}-x_{j}}{2 \beta}\right)= \begin{cases}0 & \text { if }\left|x_{i}-x_{j}\right|>\beta \\ \eta / 2 & \text { if }\left|x_{i}-x_{j}\right|=\beta \\ \eta & \text { if }\left|x_{i}-x_{j}\right|<\beta\end{cases}
$$

Applying formula (A11), we have

$$
\begin{aligned}
U\left(R_{i j}\right) & =\frac{2 \eta}{\pi} \int_{0}^{\pi / 2} \operatorname{rect}\left(\frac{R_{i j} \sin \tau}{2 \beta}\right) d \tau \\
& =\frac{4 \beta \eta}{\pi R_{i j}} \int_{0}^{\frac{R_{i j}}{2 \beta}} \frac{\operatorname{rect}(x)}{\sqrt{1-\left(\frac{2 \beta}{R_{i j}} x\right)^{2}}} d x,
\end{aligned}
$$

where $x=\frac{R_{i j} \sin \tau}{2 \beta}$. Using the definition of $\operatorname{rect}(x)$, we have

$$
U\left(R_{i j}\right)= \begin{cases}\frac{2 \eta}{\pi} \arcsin \left(\frac{\beta}{R_{i j}}\right) & \text { if } R_{i j} \geqslant \beta, \\ \eta & \text { if } R_{i j}<\beta .\end{cases}
$$

For $R_{i j} \gg \beta$, we have the long-range asymptotic behavior

$$
U\left(R_{i j}\right) \sim \frac{2 \eta \beta}{\pi} \frac{1}{R_{i j}}, \text { for } R_{i j} \gg \beta .
$$

\section{Coulomb potential}

We approach the Coulomb potential by the following type of interaction potential:

$$
V\left(x_{i}-x_{j}\right)=\frac{\beta}{\sqrt{\left(x_{i}-x_{j}\right)^{2}+\varepsilon}} \quad \text { with } \varepsilon>0 .
$$

The interaction $V\left(x_{i}-x_{j}\right)$ goes to Coulomb potential in the limit of $\varepsilon \rightarrow 0$. Applying formula (A10), we obtain

$$
\begin{aligned}
U\left(R_{i j}\right) & =\frac{\beta}{2 \pi} \int_{-\pi}^{+\pi} \frac{1}{\sqrt{R_{i j}^{2} \sin ^{2} \tau+\varepsilon}} d \tau \\
& =\frac{2 \beta}{\pi \sqrt{\varepsilon}} \int_{0}^{\pi / 2} \frac{1}{\sqrt{\varepsilon^{-1} R_{i j}^{2} \sin ^{2} \tau+1}} d \tau \\
& =\frac{2 \beta}{\pi \sqrt{\varepsilon}} K\left(i \frac{R_{i j}}{\sqrt{\varepsilon}}\right) \equiv \frac{2 \beta^{*}}{\pi} \frac{1}{\sqrt{R_{i j}^{2}+\varepsilon}} .
\end{aligned}
$$

Here, we introduced the effective coupling $\beta^{*} \equiv \beta K\left(\sqrt{\frac{R_{i j}^{2}}{R_{i j}^{2}+\varepsilon}}\right)$, where $K(x)$ is the complete elliptic integral of the first kind with the property of

$$
K(i x)=\frac{1}{\sqrt{1+x^{2}}} K\left(\sqrt{\frac{x^{2}}{x^{2}+1}}\right)
$$

for a real $x$. We use the asymptotic approximations for the first complete elliptic integral [54], i.e., $K(i x) \approx \frac{1}{x} \ln (4 x)$ for $x \gg 1$. Then we have the long-range asymptotic behavior of potential $(\mathrm{C} 3)$

$$
U\left(R_{i j}\right) \approx \frac{2 \beta}{\pi R_{i j}} \ln \left(\frac{4 R_{i j}}{\sqrt{\varepsilon}}\right), \text { for } R_{i j} \gg 1 .
$$


We see that in the limit of $\varepsilon \rightarrow 0$, where the real-space interaction $V\left(x_{i}-x_{j}\right)$ goes to Coulomb potential, the phasespace interaction potential (C4) diverges. We will analyze the physical origin of this divergence and introduce the renormalization procedure to cancel it in the next section.

\section{APPENDIX D: INVERSE POWER-LAW POTENTIAL}

We assume an interaction potential in the form of inverse power law, i.e.,

$$
V\left(x_{i}-x_{j}\right)=\frac{\beta^{2 n}}{\left|x_{i}-x_{j}\right|^{2 n}}
$$

with integers and half-integers $n \geqslant 1 / 2$. We apply the formula (A11) and obtain

$$
\begin{aligned}
U\left(R_{i j}\right)= & \frac{2 \beta}{\pi R_{i j}^{2 n}} \int_{0}^{\pi / 2} \frac{1}{\sin ^{2 n} \tau} d \tau=\frac{2 \beta}{\pi R_{i j}^{2 n}} \int_{0}^{1} \frac{1}{t^{2 n} \sqrt{1-t^{2}}} d t \\
= & \left.\frac{2 \beta}{\pi R_{i j}^{2 n}} \frac{t^{1-2 n}}{1-2 n}{ }_{2} F_{1}\left(\frac{1}{2}, \frac{1}{2}-n ; \frac{3}{2}-n ; t^{2}\right)\right|_{\varepsilon \rightarrow 0} ^{1} \\
= & \frac{2 \beta}{\pi R_{i j}^{2 n}} \frac{1}{1-2 n}\left[{ }_{2} F_{1}\left(\frac{1}{2}, \frac{1}{2}-n ; \frac{3}{2}-n ; 1\right)\right. \\
& \left.-\left.\varepsilon^{1-2 n}{ }_{2} F_{1}\left(\frac{1}{2}, \frac{1}{2}-n ; \frac{3}{2}-n ; \varepsilon^{2}\right)\right|_{\varepsilon \rightarrow 0}\right] . \quad \text { (D2) }
\end{aligned}
$$

Due to the term $\varepsilon^{1-2 n}$, the above integral diverges in the limit of $\varepsilon \rightarrow 0$ for $n>1 / 2$. We renormalize $U\left(R_{i j}\right)$ for integers $n \geqslant 1$, half-integers $n \geqslant 3 / 2$, and $n=1 / 2$ (Coulomb potential), respectively.

\section{Identities of hypergeometric function}

To continue, we should introduce some identities of hypergeometric function ${ }_{2} F_{1}(a, b ; c ; z)$ and Gamma function $\Gamma(z)$.

Identity I. Gauss's theorem [55] of hypergeometric function ${ }_{2} F_{1}(a, b ; c ; 1)$

$$
\begin{aligned}
{ }_{2} F_{1}(a, b ; c ; 1) & =\frac{\Gamma(c) \Gamma(c-a-b)}{\Gamma(c-a) \Gamma(c-b)} \text { for } \\
\operatorname{Re}[c] & >\operatorname{Re}[a+b] .
\end{aligned}
$$

Identity II. Derivatives of hypergeometric function ${ }_{2} F_{1}(a, b ; c ; z)$

$$
\begin{aligned}
\frac{d^{m}}{d z^{m}}{ }_{2} F_{1}(a, b ; c ; z) & =\frac{(a)_{m}(b)_{m}}{(c)_{m}}{ }_{2} F_{1}(a+m, b+m ; c+m ; z), \\
\text { where } \quad(q)_{m} & \equiv \frac{\Gamma(q+m)}{\Gamma(q)} .
\end{aligned}
$$

Identity III. Euler's reflection formula [56] of Gamma function

$$
\Gamma(1-z) \Gamma(z)=\frac{\pi}{\sin (\pi z)} .
$$

Identity IV. Euler's duplication formula [56] of Gamma function

$$
\Gamma(z) \Gamma\left(z+\frac{1}{2}\right)=2^{1-2 z} \sqrt{\pi} \Gamma(2 z) .
$$

Identity $V$. Taylor's expansion of Gamma function

$$
\ln \Gamma(1+z)=-\gamma z+\sum_{k=1}^{\infty} \frac{\zeta(k)}{k !}(-z)^{k} \quad \text { for }|z|<1,
$$

where $\gamma \approx 0.5772156649$ is the Euler-Mascheroni constant and $\zeta(k)$ is the Riemann zeta function at $k$. Combined with Eq. (D5), we have the following approximative identity:

$$
\Gamma(z) \approx \frac{1}{z} e^{-\gamma z} \quad \text { for }|z| \ll 1
$$

\section{Half-integers $n \geqslant 3 / 2$}

For half-integers $n=k+1 / 2$ with $k \geqslant 1$, we have the following divergence in formula (D2):

$$
\begin{aligned}
{ }_{2} & F_{1}\left(\frac{1}{2}, \frac{1}{2}-n ; \frac{3}{2}-n ; 1\right) \\
& =-\sqrt{\pi} \frac{\Gamma(k+1 / 2)}{\Gamma(k)} \frac{\sin (k \pi+\pi / 2)}{\cos (k \pi+\pi / 2)} \rightarrow \infty .
\end{aligned}
$$

It seems the phase-space potential (28) is not valid for the case of half-integers $n \geqslant 3 / 2$. However, we show this divergence is artificial and is canceled by another divergence in formula (D2). The half-integers can be approached by taking $n=k+$ $1 / 2+\epsilon$ with $\epsilon \rightarrow 0$. Then we have

$$
\begin{aligned}
& { }_{2} F_{1}\left(\frac{1}{2}, \frac{1}{2}-n ; \frac{3}{2}-n ; 1\right) \\
& \quad=\sqrt{\pi} \frac{\Gamma(k+1 / 2+\epsilon)}{\Gamma(k+\epsilon)} \frac{\cos (k \pi+\pi \epsilon)}{\sin (k \pi+\pi \epsilon)} \rightarrow \frac{1}{\epsilon} \frac{\Gamma(k+1 / 2)}{\sqrt{\pi} \Gamma(k)} .
\end{aligned}
$$

In fact, there is also a divergent term in the function of ${ }_{2} F_{1}\left(\frac{1}{2}, \frac{1}{2}-n ; \frac{3}{2}-n ; \varepsilon^{2}\right)$. To reveal it, we write the function ${ }_{2} F_{1}\left(\frac{1}{2}, \frac{1}{2}-n ; \frac{3}{2}-n ; \varepsilon^{2}\right)$ in Taylor's series using identity (D4)

$$
\begin{aligned}
{ }_{2} & F_{1}\left(\frac{1}{2}, \frac{1}{2}-n ; \frac{3}{2}-n ; \varepsilon^{2}\right) \\
& =\sum_{m=0}^{\infty} \varepsilon^{2 m} \frac{(1 / 2)_{m}(-k-\epsilon)_{m}}{k !(1-k-\epsilon)_{m}}{ }_{2} F_{1}\left(\frac{1}{2}, \frac{1}{2}-n ; \frac{3}{2}-n ; 0\right) \\
& =\sum_{m=0}^{\infty} \frac{(1 / 2)_{m}(-k-\epsilon)_{m}}{k !(1-k-\epsilon)_{m}} \varepsilon^{2 m} .
\end{aligned}
$$

We calculate the coefficient for $m=k$ in the limit of $\epsilon \rightarrow 0$

$$
\begin{aligned}
\frac{(1 / 2)_{k}(-k-\epsilon)_{k}}{(1-k-\epsilon)_{k}} \frac{1}{k !} \\
=\frac{\Gamma(k+1 / 2)}{\Gamma(1 / 2)} \frac{\Gamma(-\epsilon)}{\Gamma(-k-\epsilon)} \frac{\Gamma(1-k-\epsilon)}{\Gamma(1-\epsilon)} \frac{1}{\Gamma(k+1)} \\
=\frac{\Gamma(k+1 / 2)}{\Gamma(1 / 2)} \frac{\Gamma(-\epsilon)}{(-\epsilon) \Gamma(-\epsilon)} \frac{\Gamma(1-k-\epsilon)}{\Gamma(-k-\epsilon)} \frac{1}{\Gamma(k+1)} \\
=\frac{\Gamma(k+1 / 2)}{\Gamma(1 / 2)} \frac{1}{-\epsilon} \frac{\Gamma(1+k+\epsilon)}{\Gamma(k+\epsilon)} \\
\quad \times \frac{\sin (-k \pi-\epsilon \pi)}{\sin (\pi-k \pi-\epsilon \pi)} \frac{1}{\Gamma(k+1)}=\frac{1}{\epsilon} \frac{\Gamma(k+1 / 2)}{\sqrt{\pi} \Gamma(k)} .
\end{aligned}
$$


Therefore, this coefficient is divergent as $\epsilon \rightarrow 0$ and cancels the divergence of (D9). This means the phase-space potential (28) is still valid for half-integers $n=k+1 / 2$ with $k \geqslant 1$.

\section{Coulomb potential $n=1 / 2$}

According to identity (D3) we have

$$
{ }_{2} F_{1}\left(\frac{1}{2}, \frac{1}{2}-n ; \frac{3}{2}-n ; 1\right)=\frac{\Gamma\left(\frac{3}{2}-n\right) \Gamma\left(\frac{1}{2}\right)}{\Gamma(1-n) \Gamma(1)}=-\sqrt{\pi} \frac{\Gamma(n)}{\Gamma\left(n-\frac{1}{2}\right)} \frac{\sin (n \pi)}{\cos (n \pi)} .
$$

If we assume $n=1 / 2+\epsilon / 2$ with $\epsilon \rightarrow 0$, we have

$$
\begin{aligned}
{ }_{2} F_{1}\left(\frac{1}{2}, \frac{1}{2}-n ; \frac{3}{2}-n ; 1\right) & ={ }_{2} F_{1}\left(\frac{1}{2},-\frac{\epsilon}{2} ; 1-\frac{\epsilon}{2} ; 1\right)=\frac{\Gamma\left(1-\frac{\epsilon}{2}\right) \Gamma\left(\frac{1}{2}\right)}{\Gamma\left(\frac{1}{2}-\frac{\epsilon}{2}\right) \Gamma(1)} \\
& =\sqrt{\pi} \frac{\Gamma\left(\frac{1}{2}+\frac{\epsilon}{2}\right)}{\Gamma\left(\frac{\epsilon}{2}\right)} \frac{\cos (\pi \epsilon / 2)}{\sin (\pi \epsilon / 2)} \rightarrow \frac{1}{\sqrt{\pi}} \frac{\Gamma\left(\frac{1}{2}+\frac{\epsilon}{2}\right)}{\frac{\epsilon}{2} \Gamma\left(\frac{\epsilon}{2}\right)} \\
& =\frac{1}{\sqrt{\pi}} \frac{\Gamma\left(\frac{\epsilon}{2}\right) \Gamma\left(\frac{1}{2}+\frac{\epsilon}{2}\right)}{\frac{\epsilon}{2} \Gamma\left(\frac{\epsilon}{2}\right)^{2}}=\frac{2^{1-\epsilon} \Gamma(\epsilon)}{\frac{\epsilon}{2} \Gamma\left(\frac{\epsilon}{2}\right)^{2}} \\
& \approx 2^{1-\epsilon} \frac{\frac{1}{\epsilon} e^{-\gamma \epsilon}}{\frac{\epsilon}{2}\left(\frac{\epsilon}{2}\right)^{-2} e^{-\gamma \epsilon}}=2^{-\epsilon} \approx 1-\epsilon \ln 2
\end{aligned}
$$

Using the above formula, we have $U\left(R_{i j}\right)$ for the potential $V\left(x_{i}-x_{j}\right)=\beta\left|x_{i}-x_{j}\right|^{-1-\epsilon}$,

$$
\begin{aligned}
U\left(R_{i j}\right) & =\left.\frac{2 \beta}{\pi R_{i j}^{2 n}} \frac{t^{1-2 n}}{1-2 n}{ }_{2} F_{1}\left(\frac{1}{2}, \frac{1}{2}-n ; \frac{3}{2}-n ; t^{2}\right)\right|_{\sin \tau_{c}} ^{1} \\
& =\left.\frac{2 \beta}{\pi R_{i j}^{1+\epsilon}} \frac{1}{-\epsilon}\left(1-\epsilon \ln 2-\tau_{c}^{-\epsilon}\right)\right|_{\epsilon \rightarrow 0, \tau_{c} \ll 1} \\
& =\left.\frac{2 \beta}{\pi R_{i j}^{1+\epsilon}} \frac{1}{-\epsilon}\left(1-\epsilon \ln 2-1+\epsilon \tau_{c}^{-\epsilon} \ln \tau_{c}\right)\right|_{\epsilon \rightarrow 0, \tau_{c} \ll 1} \\
& =\left.\frac{2 \beta}{\pi R_{i j}} \ln \left(2 / \tau_{c}\right)\right|_{\epsilon=0, \tau_{c} \ll 1}
\end{aligned}
$$

[1] L. Tonks, Phys. Rev. 50, 955 (1936).

[2] M. Girardeaus, J. Math. Phys. 1, 516 (1960).

[3] E. H. Lieb, Phys. Rev. 130, 1616 (1963).

[4] E. H. Lieb and W. Liniger, Phys. Rev. 130, 1605 (1963).

[5] C. N. Yang and C. P. Yang, J. Math. Phys. 10, 1115 (1969).

[6] I. Bloch, Nat. Phys. 1, 23 (2005).

[7] I. Bloch, J. Dalibard, and W. Zwerger, Rev. Mod. Phys. 80, 885 (2008).

[8] N. Goldman et al., Rep. Prog. Phys. 77, 126401 (2014).

[9] J. Struck, C. Ölschläger, M. Weinberg, P. Hauke, J. Simonet, A. Eckardt, M. Lewenstein, K. Sengstock, and P. Windpassinger, Phys. Rev. Lett. 108, 225304 (2012).

[10] M. Olshanii, Phys. Rev. Lett. 81, 938 (1998).

[11] T. Bergeman, M. G. Moore, and M. Olshanii, Phys. Rev. Lett. 91, 163201 (2003).

[12] G. E. Astrakharchik, D. Blume, S. Giorgini, and B. E. Granger, Phys. Rev. Lett. 92, 030402 (2004).

[13] B. Paredes et al., Nature (London) 429, 277 (2004).

[14] T. Kinoshita et al., Science 305, 1125 (2004).

[15] E. Haller et al., Science 325, 1224 (2009).

[16] M. A. Cazalilla et al., Rev. Mod. Phys. 83, 1405 (2011).
[17] X.-W. Guan et al., Rev. Mod. Phys. 85, 1633 (2013).

[18] F. Calogero, J. Math. Phys. 10, 2191 (1969).

[19] Z. Idziaszek and T. Calarco, Phys. Rev. A 74, 022712 (2006).

[20] Y. Alhassid, G. F. Bertsch, and L. Fang, Phys. Rev. Lett. 100, 230401 (2008).

[21] M. Zhong-Qi and C. N. Yang, Chin. Phys. Lett. 26, 120505 (2009).

[22] X. Yin et al., Phys. Rev. A 84, 011602(R) (2011).

[23] E. J. Lindgren et al., New J. Phys. 16, 063003 (2014).

[24] T. Sowiński et al., Europhys. Lett. 109, 26005 (2015).

[25] T. Grining et al., New J. Phys. 17, 115001 (2015).

[26] H. Moritz, T. Stöferle, M. Köhl, and T. Esslinger, Phys. Rev. Lett. 91, 250402 (2003).

[27] J. Struck et al., Nat. Phys. 9, 738 (2013).

[28] M. Aidelsburger, M. Atala, S. Nascimbène, S. Trotzky, Y.-A. Chen, and I. Bloch, Phys. Rev. Lett. 107, 255301 (2011).

[29] M. Marthaler and M. I. Dykman, Phys. Rev. A 76, 010102 (2007).

[30] M. Aidelsburger, M. Atala, M. Lohse, J. T. Barreiro, B. Paredes, and I. Bloch, Phys. Rev. Lett. 111, 185301 (2013).

[31] M. Aidelsburger et al., Appl. Phys. B 113, 1 (2013). 
[32] J. H. Shirley, Phys. Rev. 138, B979 (1965).

[33] H. P. Breuer and M. Holthuas, Ann. Phys. (NY) 211, 249 (1991).

[34] A. Buchleitner, D. Delande, and J. Zakrzewski, Phys. Rep. 368, 409 (2002).

[35] S. Sauer, F. Mintert, C. Gneiting, and A. Buchleitner, J. Phys. B 45, 154011 (2012).

[36] M. Grifoni and P. Hänggi, Phys. Rep. 304, 229 (1998).

[37] N. H. Lindner, G. Refael, and V. Galitski, Nat. Phys. 7, 490 (2011).

[38] A. Gómez-León and G. Platero, Phys. Rev. Lett. 110, 200403 (2013).

[39] G. Usaj, P. M. Perez-Piskunow, L. E. F. Foa Torres, and C. A. Balseiro, Phys. Rev. B 90, 115423 (2014).

[40] Z. Gu, H. A. Fertig, D. P. Arovas, and A. Auerbach, Phys. Rev. Lett. 107, 216601 (2011).

[41] S. Andre, L. Guo, V. Peano, M. Marthaler, and G Schön, Phys. Rev. A 85, 053825 (2012).

[42] M. S. Rudner, N. H. Lindner, E. Berg, and M. Levin, Phys. Rev. X 3, 031005 (2013).

[43] L. Guo and M. Marthaler, New J. Phys. 18, 023006 (2016).
[44] L. Guo, M. Marthaler, and G. Schön, Phys. Rev. Lett. 111, 205303 (2013).

[45] S. N. Rasband, in Chaotic Dynamics of Nonlinear Systems (Wiley, New York, 1990), pp. 92-95.

[46] R. L. Devaney, An Introduction to Chaotic Dynamical Systems, 2nd ed. (Westview Press, Boulder, CO, 2003).

[47] T. Bilitewski and N. R. Cooper, Phys. Rev. A 91, 033601 (2015).

[48] S. Choudhury and E. J. Mueller, Phys. Rev. A 91, 023624 (2015).

[49] P. Jung, S. Butz, M. Marthaler, M. V. Fistul, J. Leppäkangas, V. P. Koshelets, and A. V. Ustinov, Nat. Commun. 5, 3730 (2014).

[50] L. S. Brown and G. Gabrielse, Rev. Mod. Phys. 58, 233 (1986).

[51] W. Paul, Rev. Mod. Phys. 62, 531 (1990).

[52] D. Leibfried, R. Blatt, C. Monroe, and D. Wineland, Rev. Mod. Phys. 75, 281 (2003).

[53] R. Blatt and D. J. Wineland, Nature (London) 453, 1008 (2008).

[54] D. Karpa and S. M. Sitnikb, J. Comput. Appl. Math. 205, 186 (2007).

[55] L. J. Slater, Generalized Hypergeometric Functions (Cambridge University Press, Cambridge, UK, 1966).

[56] http://mathworld.wolfram.com/ReflectionRelation.html 